\title{
Sensitivity of lateral heat transfer on the convection onset in a transient Rayleigh-Bénard-Marangoni flow
}

\author{
Louis-Henri Baudey-Laubier, Benoît Trouette $\bowtie$ Eric Chénier \\ \{louis-henri.baudey-laubier; benoit.trouette;eric.chenier\}@u-pem.fr \\ MSME Laboratoire de Modélisation et Simulation Multi Echelle, \\ UMR 8208 CNRS, Université Paris-Est Marne-la-Vallée, \\ 5 Boulevard Descartes, 77454 Marne-la-Vallée Cedex 2, FRANCE
}

Version: April 17, 2018

\section{Contents}

1 Introduction

2 Physical, mathematical and numerical models 3

3 Results 4

3.1 Comments on the convection onset for a transient solution . . . . . . . . . . . . . . . . . . 5

3.2 Physical criterion for the diffusion $/$ convection transition . . . . . . . . . . . . . . . . . . . . 6

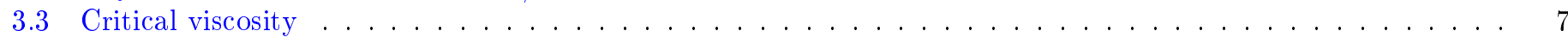

3.4 Spatio-temporal diagrams: physical versus numerical disturbances . . . . . . . . . . . . . . 8

3.5 Propagation celerity of the convection wave . . . . . . . . . . . . . . . . .

3.6 Critical time for the convection onset . . . . . . . . . . . . . . . . . . . . . . 10

3.7 Convection cell characterization . . . . . . . . . . . . . . . . . . . . . . . . 11

3.8 Perturbations induced at the initial time . . . . . . . . . . . . . . . . . . . . . 12

4 Summary and concluding remarks 13

\begin{abstract}
The convection onset in a evaporation thermal model of a solute/solvent solution is numerically studied. The transient nature of the diffusion temperature profile requires to control the perturbations which will lead to the transition to a convective flow. Contrary to previous papers, we adopted here a perturbation mode, which is physical and experimentally reproducible, by accounting for a heat transfer at the lateral walls. Based on the Péclet number evaluated in the core flow field only, the critical viscosities and the corresponding times for the transition between a diffusion and convection regimes were calculated as a function of the fluid layer thickness, the aspect ratio and the convective heat transfer coefficient. The study of the spatio-temporal diagrams allowed us to characterize the way the convection propagates in the fluid domain as well as the evolution of the mean cell size and wavenumber.
\end{abstract}

Keywords: Rayleigh-Bénard-Marangoni instabilities; numerical simulations; transient flow; evaporation model;

\section{Introduction}

Many industrial processes such as painting, inking or packaging involve the drying of binary liquid films with a least one volatile component. For a solute/solvent solution brought in contact with a gas environment at standard conditions, the solvent vaporization at the liquid/gas interface leads to an unsteady heat and mass transfer into the whole liquid layer. The solvent evaporation at the liquid/gas interface creates, at the same time, a decrease in the solvent concentration, a cooling due to the phase change endothermic phenomenon, and a reduction in the solution layer thickness. These thermal and solutal variations at the interface then diffuse into the fluid layer. Under specific conditions, this transient diffusive regime can turn out to be unstable because of the buoyancy and/or surface tension forces induced by the thermal and/or solutal gradients. The slow decrease in the fluid layer thickness, combined with the viscosity increasing produced by the enriched solution in

corresponding author 
solute, implies the convective flow must always fades away over the long time, so that the transfer evolution ends up being driven by the diffusion, again.

The mechanisms leading to natural convective flows have been widely studied. Amongst the earlier works upon convective instabilities, we can mention the theoretical contribution by Pearson [17] for surface tension driven flows (Bénard-Marangoni instabilities) and the experiments by Blair and Quinn [4] for the buoyancy-driven flows (Rayleigh-Bénard instabilities). A recent numerical, experimental and theoretical review involving surface tension instabilities has been published by Wang et al. [22] to elucidate the mechanisms of the Marangoni effect.

The models used to study the film or droplet evaporation introduce different levels of descriptions. For example, the evaporation of a pure fluid involves the temperature variable, while the concentration of the components must also be taken into account for mixtures. The transfers at the interface are also the subject of numerous models. They can result from the full simulation of the heat and fluid flow in the gaseous phase, or be determined by assuming a diffusion regime in the environment, or more simply be modelled via Newton's laws and global transfer coefficients. The choice for the model of the interface deformation depends greatly on the studied problem, too. It can be rigid and motionless if the evaporated liquid volume is negligible, moveable but non-deformable to account for volume loss, or completely deformable when its shape results from the stress jumps and the continuity of the velocity. The dynamic of solutions may converge toward a steady or quasi-steady flow, or evolve continuously as a function of time, depending on the applied boundary conditions or the temporal range of interest. The consequences upon numerical methods for the search of the convection onset are important since the classical linear stability analysis is a priori not applicable when the diffusive solution is unsteady.

In the evaporation case of pure volatile liquids, the experimental works by Colinet et al. [6] and Mancini and Maza [15] focused on the coupling between the solution and the surrounding gas phase, while numerical studies were conducted by Merkt and Bestehorn [16]. They showed that, for moderate evaporation rates, the one-sided layer model where only the liquid film is simulated is a good model for real problems. In that case, a generalized heat transfer coefficient accounting for both the convective heat transfer and the thermal evaporation flux is introduced at the liquid/gas interface. In this scope, Machrafi et al. [14] developed a one-sided model to study the thermal Marangoni instability produced by a liquid evaporation in an inert gas. The use of a linear stability analysis of the transient base state using the frozen time approach showed the conditions for which the introduction of a generalized heat transfer coefficient, function of the perturbation wavenumber, is particularly relevant at the interface.

In the case of evaporation of a solute/solvent solution, both thermal and solutal contributions are able to destabilize the diffusive solution. In experimental studies by Bassou and Rharbi [1], the authors highlighted the correlation between the solutal Bénard-Marangoni convection cell size and the size of the surface corrugations visible on the deposit at the end of the drying process. For a water/ethanol solution, Machrafi et al. [12] have numerically studied the buoyancy-surface tension coupling. In a quasi-steady regime, the authors developed linear stability analysis to obtain the neutral stability curves as a function of the solutal/thermal Marangoni/Rayleigh numbers. Later on, they carried on their works with transient solutions and the frozen-time approach [13], what allowed them to compute the neutral stability curves in the parameter plane spanned by the liquid layer thickness and the elapsed time after the exposure of the aqueous solution to the surrounding gas.

The present contribution is a part of a more general study devoted to the understanding of the fluid flow and heat and mass transfer in a specific solution of Polyisobutylene and Toluene in which the viscosity strongly depends on the solute concentration. The first work was conducted by Toussaint et al. [19] with experiments. They interested in the convection onset for wide ranges of initial liquid layer thicknesses and solute concentrations (viscosities). They also studied the lifetime of convection cells and the time from which the viscous skin is created at the fluid surface. Based on these experimental results and considering the very large difference in the thermal and solutal diffusion time scales, two evaporation models have been worked out. One is valid at the first beginning of the drying and rests on a uniform concentration field, while the second model is valid later and assumes a thermal equilibrium in the whole fluid layer. The study of the solutal model was carried out by Trouette et al. [21]. The transition from the diffusive state to a convective flow was studied with transient numerical simulations, and the preponderance of the solutal Marangoni convection over the buoyancy one was shown. Recently, Yiantsios et al. [23] performed numerical simulations of the solutal Bénard-Marangoni convection, considering both the strong dependence of viscosity with the solute concentration and the free surface deformation. They showed that the wrinkles observed by Bassou and Rharbi [1] at the end of the drying may be viewed as the signature of the convection cells. The transient thermal model was studied successively by Touazi et al. [18] and Trouette et al. [20], who carried out non-linear numerical simulations in two or three-dimensional geometries, applying random perturbations on some initial fields (velocity [18] and temperature [20]) in order to control the convection onset. The viscosity thresholds were obtained for a wide range of fluid layer thicknesses and these results turned out to be in qualitative agreement with the experimental data by Toussaint et al. [19]. The linear stability analysis by Doumenc et al. [7], performed with the non normal approach, provided threshold values in the same order of magnitude than those obtained with the non-linear simulations and the experimental works [18, 19, 20]: for transient problems and a given layer thickness, the diffusive and convective transition viscosity is not clearly defined, as for steady base flows, but by a blurred interval depending essentially on the amplitude of the initial perturbation and the used criterion to delimit the diffusion and convection regimes. An overview upon some of 
these recent works, including the thermal and solutal models, can be found in [8].

The former works on Polyisobutylene/Toluene solutions rely on a control of the convection onset through random disturbances applied at the initial time. One difficulty raised by this approach is how to choose physically the magnitude, and in a lesser extend, the shape of the initial perturbations. Furthermore, the introduction of uncertainties in the numerical model makes the reproducibility of the simulation results tricky, except if the perturbation field is fully known on a given mesh, and if the same numerical code is used; otherwise, the solution risks to be modified. As a consequence, this approach may raise the issue of the convergence of the numerical solutions as a function the mesh size, when the disturbance is mesh dependent. In this contribution, we consider a thermal model for the evaporation of a Polyisobutylene/Toluene solution. The perturbations of the diffusion transient solution, which give rise to the convection onset, are physically caused by the convective heat transfer with the surrounding gas modelled at the lateral boundaries. The rest of the paper is organized into three sections. The first one describes the physical, mathematical and numerical models. The next section is devoted to the result presentation. After a short introduction and discussion upon the transient solutions and the used criterion to define the transition between the diffusion and convection regimes, the critical viscosities are given as a function of the layer thickness. The accurate description of the convective cell appearance, evolution and their fade-out is then proposed on the basis of spatio-temporal diagrams. Some comparisons are also provided using simulations with disturbances at the initial conditions. The last section is devoted to the concluding remarks and a placement in perspective of our threshold viscosities and those of the literature.

\section{Physical, mathematical and numerical models}

A homogeneous Polyisobutylene/Toluene (PIB/Toluene) solution at rest is contained into an open container topped by air at standard atmospheric conditions. In contact with the surrounding gas, the liquid solvent evaporates. As evaporation is an endothermic process, some amount of latent heat is taken away from the solution at the gas/liquid interface. Subsequently, the cooling and the solvent deficit are diffusing into the fluid layer thickness. Under specific dimensionless parameters (to be determined), the transient diffusive solution becomes unstable and a fluid motion occurs. This instability may be driven by surface tension and/or density variations, each of them induced by thermal or solutal inhomogeneities. As the physical approximations have been widely discussed in previous papers [7, 8, 18, 19, 20], only the main assumptions are here reminded for sake of brevity. A one-sided model is used and the heat transfer with the surrounding gas is taken into account by a constant and uniform convective coefficient. Since the mass diffusivity of the PIB/toluene solution is about three order of magnitude smaller than its thermal diffusivity, it is possible to identify a thermal regime for short times where the solvent fraction is assumed uniform in the entire liquid film [3, 8]. From this assumption, it results that both the physical properties and the fluid thickness do not evolve substantially: they are kept constant at their initial values. Lastly and in accordance with the previous works [18, 20], a two-dimensional approximation is used since the transitions from diffusion solutions to convection flows do not depend significantly on the space dimension. Therefore, the fluid layer consists of a rectangular domain of aspect ratio $A=L / e$, where $L$ is the horizontal length and $e$ the thickness of the layer. Thus, the heat transfer and fluid flow are governed by the Navier-Stokes and energy equations, expressed with the Boussinesq approximation.

Based on the fluid thickness $e$, the thermal diffusivity $\alpha$ and the density $\rho$, the velocity, time and pressure scales are respectively defined by $\alpha / e, e^{2} / \alpha$ and $\rho \alpha^{2} / e^{2}$. Let us first note $H=h+\left.L_{\mathrm{vap}}\left(\mathrm{d} \Phi_{s} / \mathrm{d} T\right)\right|_{T_{0}}$, with $h$ the convective heat transfer coefficient at the free surface, $\Phi_{s}$ the mass flux density, $L_{\mathrm{vap}}$ the specific latent heat and $T_{0}$ the temperature of the surrounding gas. The reduced temperature difference then writes $\Theta=\left(T-T_{0}\right) / \Delta T$, where $\Delta T=L_{\text {vap }} \Phi_{s} / H$ is the difference between the uniform temperature at the final steady state and $T_{0}$. The governing dimensionless equations for the fluid flow and the heat transfer in the liquid film read: $\forall(x, y) \in] 0, A[\times] 0,1[$

$$
\begin{gathered}
\nabla \cdot \mathbf{V}=0 \\
\frac{\partial \mathbf{V}}{\partial t}+(\mathbf{V} \cdot \nabla) \mathbf{V}=-\nabla P+\operatorname{Pr} \nabla^{2} \mathbf{V}+\operatorname{RaPr} \Theta \mathbf{e}_{y} \\
\frac{\partial \Theta}{\partial t}+(\mathbf{V} \cdot \nabla) \Theta=\nabla^{2} \Theta
\end{gathered}
$$

where $\mathbf{V}=U \mathbf{e}_{x}+V \mathbf{e}_{y}$ and $P$ are the dimensionless velocity and pressure fields. 
The dimensionless set of boundary conditions are expressed as follows:

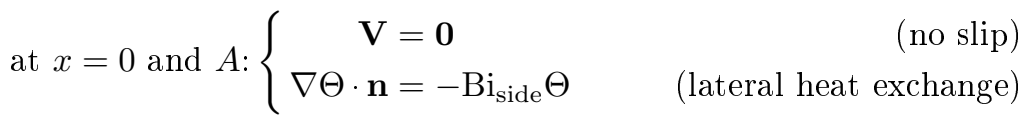

$$
\begin{aligned}
& \text { at } y=0:\left\{\begin{aligned}
\mathbf{V} & =\mathbf{0} \\
\frac{\partial \Theta}{\partial y} & =0
\end{aligned}\right. \text { (adiabatic surface) } \\
& \text { at } y=1:\left\{\begin{array}{rlrl}
\frac{\partial U}{\partial y} & =-\mathrm{Ma} \frac{\partial \Theta}{\partial x} & & \text { (thermocapillary stress) } \\
V & =0 & & \text { (zero mass flux, see [7]) } \\
\frac{\partial \Theta}{\partial y} & =-\operatorname{Bi}(\Theta+1) & \text { (energy balance) }
\end{array}\right.
\end{aligned}
$$

The dimensionless parameters are the Rayleigh, Prandtl, Marangoni and Biot numbers, as well as the aspect ratio $A=L / e$. They are defined as $\mathrm{Ra}=g \beta \Delta T e^{3} /(\nu \alpha), \operatorname{Pr}=\nu / \alpha$ and $\mathrm{Ma}=-\left.(\mathrm{d} \gamma / \mathrm{d} T)\right|_{T_{0}} \Delta T e /(\rho \nu \alpha)$, with $g$ the gravity acceleration, $\beta$ the thermal expansion isochoric coefficient, $\nu$ the kinematic viscosity and $\gamma$ the surface tension. The Biot numbers at the top interface and at the lateral sides are $\mathrm{Bi}=H e / k$ and $\mathrm{Bi}_{\text {side }}=h_{\text {side }} e / k$, where $k$ is the thermal conductivity and $h_{\text {side }}$ is the convective heat transfer coefficient along the vertical walls. For thermally isolated lateral surfaces, $h_{\text {side }}$ is set to zero. The fluid flow is initialized at rest with small temperature fluctuations around the surrounding gas temperature:

$$
\text { for } t=0, \forall(x, y) \in] 0, A[\times] 0,1\left[:\left\{\begin{array}{l}
\mathbf{V}=\mathbf{0} \\
\Theta=r \times \xi(x, y) \quad \text { (except otherwise mentioned, } r=0 \text { ) }
\end{array}\right.\right.
$$

where $r$ is the dimensionless amplitude of the perturbation and $\xi$ a probabilistic function drawn from the standard uniform distribution in the range $]-0.5,0.5]$. If $r=0$, the initial temperature is homogeneous, equal to zero.

The governing equations (1), with the boundary conditions (2) and the initial conditions (3), are discretized with a spatial and temporal second order finite volume scheme on a staggered grid. The temporal approximations rely on the Gear scheme, with an explicit Adams-Bashforth method for the velocity and temperature advection terms. The velocity and pressure fields are uncoupled with the Goda's algorithm [9]. Additional details on the numerical method and the validations are provided in [3].

\section{Results}

Except for the dynamic viscosity, all the other physical properties of the solution are assumed equal to those of the pure solvent: $k=0.142 \mathrm{~W} /(\mathrm{m} \cdot \mathrm{K}), c_{p}=1710 \mathrm{~J} /(\mathrm{kg} \cdot \mathrm{K}), \rho=866 \mathrm{~kg} / \mathrm{m}^{3}, \beta=1.07 \times 10^{-3} \mathrm{~K}^{-1}, L_{\text {vap }}=396 \mathrm{~kJ} / \mathrm{kg}, \mathrm{d} \gamma /\left.\mathrm{d} T\right|_{T_{0}}=$ $-11.9 \times 10^{-5} \mathrm{~N} /(\mathrm{m} \cdot \mathrm{K}), h=28 \mathrm{~W} /\left(\mathrm{m}^{2} \cdot \mathrm{K}\right)$ and $\alpha=9.59 \times 10^{-8} \mathrm{~m}^{2} / \mathrm{s}$. Unless otherwise stated, the lateral heat transfer coefficient is set to $h_{\text {side }}=10 \mathrm{~W} /\left(\mathrm{m}^{2} \cdot \mathrm{K}\right)$. The fluid viscosity is a free parameter since it depends on the PIB volume fraction $\varphi_{\text {PIB }}$ used to prepare the mixture: $\log _{10}(\mu)=8.235+14.02 X+6.575 X^{2}+1.392 X^{3}+0.1114 X^{4}$ with $X=\log _{10}\left(\varphi_{\mathrm{PIB}}\right)[18$, 19].

Since the main fluid properties are fixed, the dimensionless parameters depend only on the fluid thickness and the viscosity. With $e$ in $\mathrm{mm}$ and $\mu$ in $\mathrm{mPa} \cdot \mathrm{s}$, the dimensionless parameters are then defined by

$$
\left(\mathrm{Ra}, \mathrm{Ma}, \mathrm{Pr}, \mathrm{Bi}, \mathrm{Bi}_{\text {side }}\right)=\left(451 e^{3} / \mu, 5851 e / \mu, 12 \mu, 0.2 e, 0.07 e\right)
$$

Therefore, for the sake of simplicity, most results will be discussed and analysed as a function of two physical parameters only, the thickness $e$ and the dynamic viscosity $\mu$ of the fluid.

In the next sections, three fluid layer thicknesses are studied to scan the different kinds of convection flows, namely $e=1 \mathrm{~mm}$ for the capillary driven flow (Bénard-Marangoni convection), $e=8 \mathrm{~mm}$ for the coupled capillary and buoyancy driven flow (Rayleigh-Bénard-Marangoni convection) and $e=20 \mathrm{~mm}$ for the buoyancy driven flow (Rayleigh-Bénard convection) [18].

The sensitivity of the numerical parameters was carefully checked for the different thicknesses [3]. The time steps are chosen equal to $\Delta t=10^{-3}, 10^{-4}$ and $4 \times 10^{-5}$ for $e=1,8$ and $20 \mathrm{~mm}$, respectively. A sufficient number of control volumes across the fluid thickness was shown to be $N_{y}=40$, the mesh size is then defined by $\left(N_{x}, N_{y}\right)=(40 A, 40)$ with $A$ the aspect ratio. For the capillary driven flows $\left(e=1\right.$ and $8 \mathrm{~mm}$ ), a geometric refinement with the scale factor $C_{y}=0.95$ is applied in the vertical direction to capture efficiently the capillary gradients at the upper free surface. 


\subsection{Comments on the convection onset for a transient solution}

For adiabatic walls $\left(\mathrm{Bi}_{\text {side }}=0\right.$ in Eq. $\left.(2 \mathrm{a})\right)$, the convection onset is directly driven by the thermal gradients created by the evaporation process. Thus, if these gradients are too small, no fluid motion will take place and the solution will remain purely diffusive. To highlight the evolution of the vertical gradient, the temperature difference between the bottom and top surfaces of the fluid layer is drawn as a function of time for the one-dimensional unsteady pure diffusive problem, and for three thicknesses (Fig. 1). The curve evolutions clearly show that the temperature difference, and then the vertical thermal

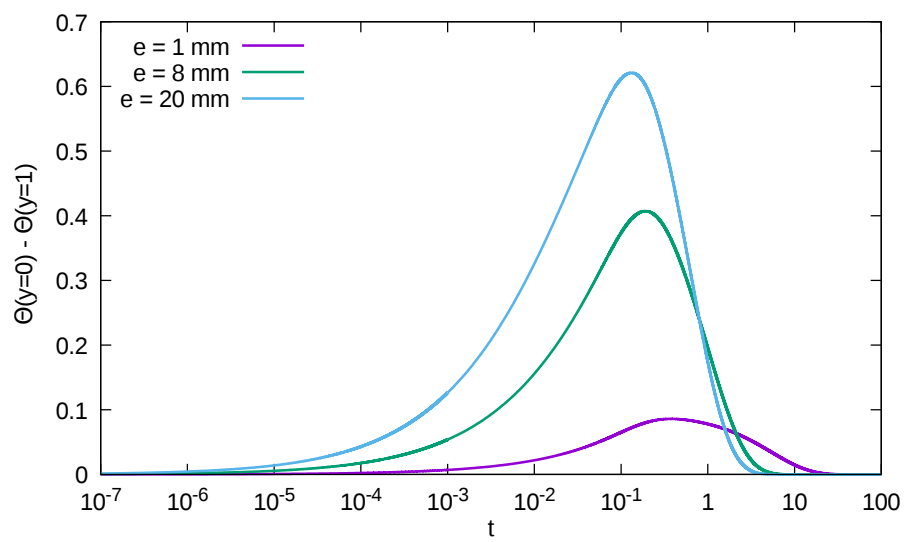

Figure 1: Evolution of the diffusion temperature difference $\Delta \Theta_{\max }=\Theta(y=0)-\Theta(y=1)$ for three thicknesses, $e=1,8$ and $20 \mathrm{~mm}$.

gradient, are transient. Therefore, the velocity field can grow or be sustained only during a finite time range where the temperature difference is large enough. Past this temporal interval, the thermal and viscous diffusions dominate upon the buoyant and/or capillary convections, so that the fluid flow is damped out and ends up to die out.

In the linear stability analysis framework, the convection onset is due to the growth of a small disturbance. For a steady base flow, there is a unique time scale which corresponds to the inverse of the perturbation growth rate. Assuming a unique attractor in the phase space, the asymptotic flow won't depend on the shape of the perturbation, nor on the positive value of its growth rate. On the other hand, if the base solution is transient (as it is shown in Fig. 1), a competition between the time scale of the perturbation and the time scale of the base flow evolution occurs. It results that the appearance of the convection becomes dependent of the magnitude of the perturbation: a too small initial perturbation won't be able to create a significant velocity flow over the time scale of the base solution.

For adiabatic walls $\left(\mathrm{Bi}_{\text {side }}=0\right.$ in Eq. (2a)) and a uniform initial temperature field $(r=0$ in Eq. (3)), the destabilization is then necessarily numerical in its origin. It is, for example, due to the number of significant digits, the accuracy of the linear solvers, ill-conditioned matrices, the Navier-Stokes solver, ... Therefore, improving the overall accuracy of the numerical scheme will shift the transition threshold towards higher Rayleigh and Marangoni numbers. Obviously, this shifting in the parameter values has no physical meaning and must be avoided. Illustrations and a more thoroughly discussion about this issue are provided in Sec. 3.4. In order to be free from this constraint, two different options are chosen in this work:

(i) either the intensity of the initial temperature disturbance is controlled through the small parameter $r>0$ (see the initial conditions (3)) with adiabatic lateral walls $\left(\mathrm{Bi}_{\text {side }}=0\right.$ in Eq. (2a)),

(ii) or the initial temperature field is kept uniform $(r=0$ in Eq. (3)), but the lateral walls are submitted to a convective heat transfer driven by a Biot number Bi $\mathrm{side}_{\mathrm{s}}>0$ in Eq. (2a).

The former approach based on random perturbations applied on a initial field was already considered in order to identify the convection onset in a PIB/toluene solution for the thermal [18] and solutal [8, 21] regimes in two-dimensional models. This idea was also extended by Chénier et al. [5] and Trouette et al. [20] in the thermal regime, using more sophisticated perturbations based on spatial auto-correlations principles. Although these authors concluded that neither the nature nor the amplitude of the initial perturbation acts significantly on the convection onset, the physical meaning of these perturbations remains questionable. Numerous other choices for the perturbations could have been adopted. For capillary driven flows, spatial disturbances may be applied to the gas temperature surmounting the fluid solution. This kind of perturbation is all the more relevant that the lateral confinement effects are negligible, as it occurs for the evaporation of a fine fluid layer in large dishes. However, the spatial shape and the temporal evolution of the fluctuations are a priori unknown. Since there is no suitable experimental data, randomly disturbances in space and time should have been chosen. But as mentioned before, this choice is physically questionable again. To overcome this issue, the latter approach is proposed in this work. The 'perturbation' of the one dimensional transient diffusion profile is produced by means of a convective heat transfer at the lateral walls. Physically, this amounts to considering non-adiabatic vertical walls. Indeed, because of the evaporation at the free surface, the liquid temperature becomes less than the surrounding air temperature. The small horizontal thermal gradients, 
which set up along the container vertical walls, produces a local fluid motion due to the buoyancy and the temperature dependent surface tension. According to whether the core flow is linearly stable or not, these localized disturbances will be damped or amplified to possibly create a convection flow in the whole fluid layer.

\subsection{Physical criterion for the diffusion/convection transition}

The transition between a diffusive solution and a convective flow is measured by the Péclet number based on the maximal velocity magnitude and the fluid thickness. To free from the fluid motion induced by the thermal gradients close to the lateral walls, the evaluation of the Péclet number is restricted to the core fluid domain of width $2 e$, defined by the rectangle $(x-A / 2, y) \in[-1,1] \times[0,1]:$

$$
\operatorname{Pe}_{\text {core }}(t)=\max _{\substack{(x-A / 2, y) \in \\[-1,1] \times[0,1]}}\|\mathbf{V}(x, y ; t)\|
$$

The solution will be considered to be convective if the core Péclet number $\mathrm{Pe}_{\text {core }}(t)$ exceeds temporarily the unity in time. Inversely, the solution is said diffusive if $\mathrm{Pe}_{\text {core }}(t)<1$, for all $t$. Two characteristic times can then be identified depending on whether the solution becomes convective $\left(t_{\mathrm{d} / \mathrm{cv}}\right)$ or get back to a diffusive regime $t_{\mathrm{cv} / \mathrm{d}}($ see Fig. 2$)$ :

$$
\begin{aligned}
& \exists t_{\mathrm{d} / \mathrm{cv}}>0 \text { such that } \operatorname{Pe}_{\mathrm{core}}\left(t_{\mathrm{d} / \mathrm{cv}}\right)=1 \text { and } \forall t<t_{\mathrm{d} / \mathrm{cv}}, \operatorname{Pe}_{\text {core }}(t)<1 \\
& \exists t_{\mathrm{cv} / \mathrm{d}}>0 \text { such that } \operatorname{Pe}_{\mathrm{core}}\left(t_{\mathrm{cv} / \mathrm{d}}\right)=1 \text { and } \forall t>t_{\mathrm{cv} / \mathrm{d}}, \operatorname{Pe}_{\text {core }}(t)<1
\end{aligned}
$$

The critical parameters $e_{\mathrm{c}}$ and $\mu_{\mathrm{c}}$ are defined when the both criteria $(6 \mathrm{a})$ and $(6 \mathrm{~b})$ are satisfied at the same time $t_{\mathrm{c}}$ :

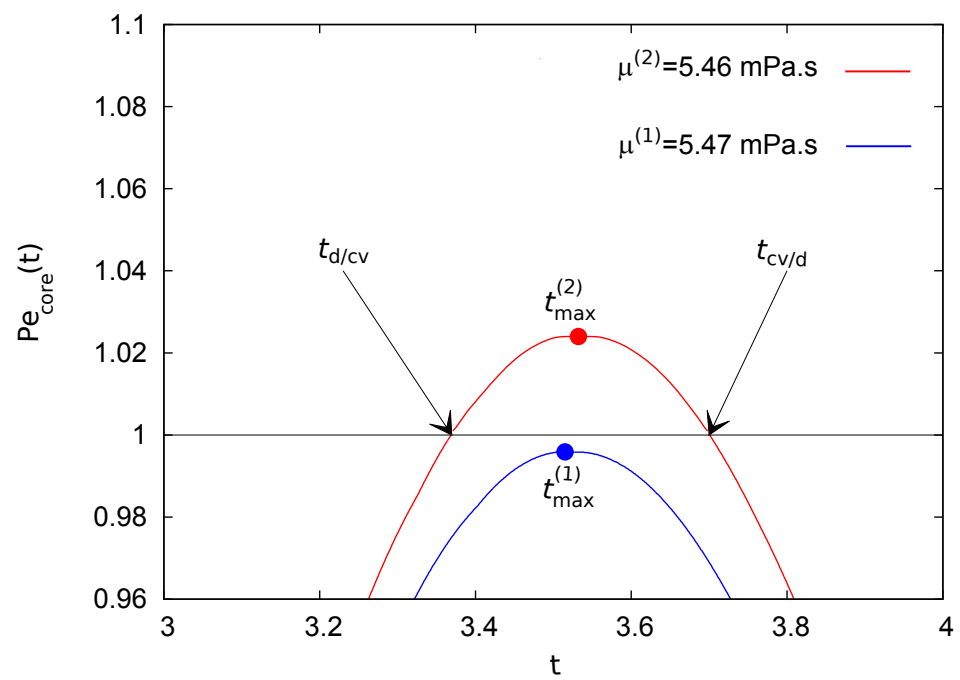

Figure 2: Core Péclet evolution $\mathrm{Pe}_{\text {core }}(t)$ for two viscosities, $e=1 \mathrm{~mm}, h_{\text {side }}=10 \mathrm{~W} /\left(\mathrm{m}^{2} \cdot \mathrm{K}\right)$ and $r=0$.

$$
\exists t_{\mathrm{c}}>0 \text { such that } \mathrm{Pe}_{\text {core }}\left(t_{\mathrm{c}}\right)=1 \text { and } \forall t \neq t_{\mathrm{c}}, \mathrm{Pe}_{\mathrm{core}}(t)<1
$$

and consequently $t_{\mathrm{c}}=t_{\mathrm{d} / \mathrm{cv}}=t_{\mathrm{cv} / \mathrm{d}}$.

For a given fluid thickness $e$, an approximation of the critical viscosity $\mu_{\mathrm{c}}$ that allows us to get the condition (7) is searched. At $\mu=\mu_{\mathrm{c}}, e$ is the critical thickness $e_{\mathrm{c}}$. From a practical point of view, the critical value $\mu_{\mathrm{c}}$ is evaluated thought an iterative procedure. As a first step, rough lower and upper bounds of $\mu_{\mathrm{c}}$ with two viscosities is sought: the viscosity $\mu^{(1)}$ is such that the solution remains purely diffusive $\left(\forall t>0, \mathrm{Pe}_{\text {core }}(t)<0\right)$ and the viscosity $\mu^{(2)}<\mu^{(1)}$ is such that conditions $(6)$ are fulfilled. The time $t_{\max }^{(1)}$ (resp. $t_{\max }^{(2)}$ ), which corresponds to the maximal value of the core Péclet evolution $\mathrm{Pe}_{\mathrm{core}}^{(1)}(t)$ for $\mu^{(1)}$ (resp. $\mathrm{Pe}_{\text {core }}^{(2)}(t)$ for $\left.\mu^{(2)}\right)$, is taken in order to proceed to a new approximation of $\mu_{\mathrm{c}}$ via the false position method:

$$
\text { do while } 2 \frac{\mu^{(1)}-\mu^{(2)}}{\mu^{(1)}+\mu^{(2)}}>\varepsilon_{\mu}, \quad\left\{\begin{array}{l}
\tilde{\mu}_{\mathrm{c}}=\frac{\left[\mathrm{Pe}_{\text {core }}^{(2)}\left(t_{\max }^{(2)}\right)-1\right] \times \mu^{(1)}+\left[1-\mathrm{Pe}_{\text {core }}^{(1)}\left(t_{\max }^{(1)}\right)\right] \times \mu^{(2)}}{\operatorname{Pe}_{\text {core }}^{(2)}\left(t_{\text {max }}^{(2)}\right)-\mathrm{Pe}_{\text {core }}^{(1)}\left(t_{\text {max }}^{(1)}\right)} \\
\text { if } \operatorname{Pe}_{\text {core }}\left(\tilde{\mu_{c}}, t\right)<1 \text { then } \\
\mu^{(1)} \leftarrow \tilde{\mu}_{\mathrm{c}} \\
\text { else } \\
\mu^{(2)} \leftarrow \tilde{\mu}_{\mathrm{c}}
\end{array}\right.
$$

In this algorithm, the stopping criteria is set to $\varepsilon_{\mu}=10^{-2}$. This method turns out to be usually convergent if the initial 
range of viscosity is not too large. When the convergence is reached, the critical value $\mu_{\mathrm{c}}$ is approximated by the updated $\tilde{\mu}_{\mathrm{c}}$ calculated from the linear combination of the last viscosities $\mu^{(1)}$ and $\mu^{(2)}$.

\subsection{Critical viscosity}

The convection onset in the core fluid layer is illustrated in Fig. 3, and it is due to the following phenomena. The thermal flux,

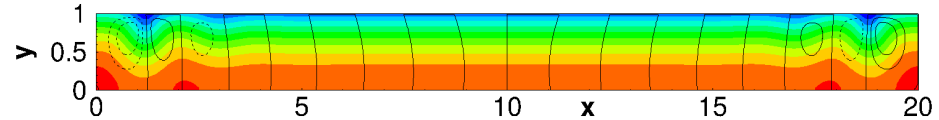

(a) $t=0.5, \Theta_{\mathrm{av}}=-0.08885$

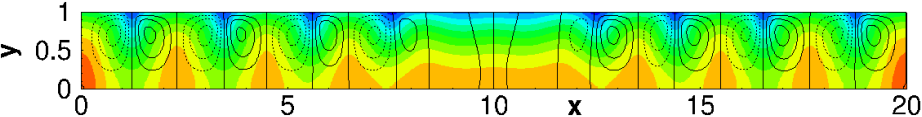

(b) $t=1, \Theta_{\mathrm{av}}=-0.1695$

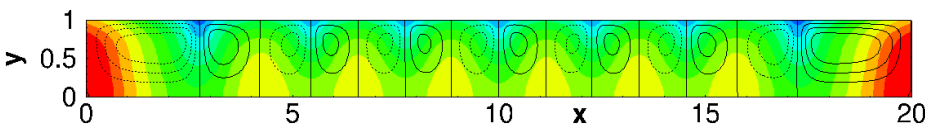

(d) $t=3, \Theta_{\mathrm{av}}=-0.4266$

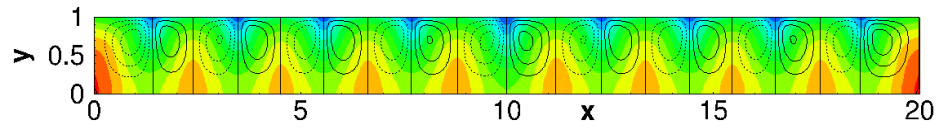

(c) $t=1.5, \Theta_{\mathrm{av}}=-0.2435$

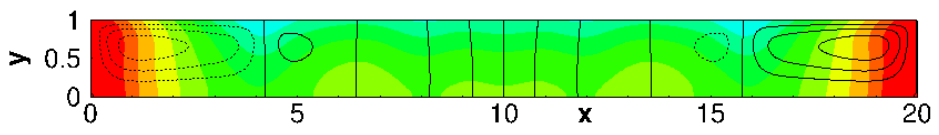

(e) $t=6, \Theta_{\mathrm{av}}=-0.6652$

Figure 3: Fluctuation of the temperature field around its average value $\Theta_{\text {av }}(t)$ (coloured field, $\Theta(x, y ; t)-\Theta$ av $(t) \in$ $[-0,06 ; 0,04])$ and stream lines for $e=1 \mathrm{~mm}, \mu=3.25 \mathrm{mPa} \cdot \mathrm{s}, h_{\text {side }}=10 \mathrm{~W} /\left(\mathrm{m}^{2} \cdot \mathrm{K}\right)$ and $r=0$.

which originates from the convective heat transfer at the lateral walls, creates local temperature perturbations to the one dimensional transient diffusive state. Because of the resulting buoyancy and surface tension inhomogeneities, weak flows in the vicinity of the vertical surfaces develop (Fig. 3(a)). If the core fluid is enough unstable, the local disturbances are amplified in time and spread in space (Figs. 3(a)-3(b)), to finally invade the whole fluid layer (Figs. 3(c)-3(d)), before being damped out for the long times in the central region (Fig. 3(e)). Obviously, the ability of the flow to maintain or amplify the convection depends on the effective Rayleigh and Marangoni numbers which evolve with the local temperature difference (see Fig. 1).

In figure 4 are reported the viscosity thresholds $\mu_{\mathrm{c}}$ for $e=1 \mathrm{~mm}, 8 \mathrm{~mm}$ and $20 \mathrm{~mm}$ as a function of the aspect ratio $A$.

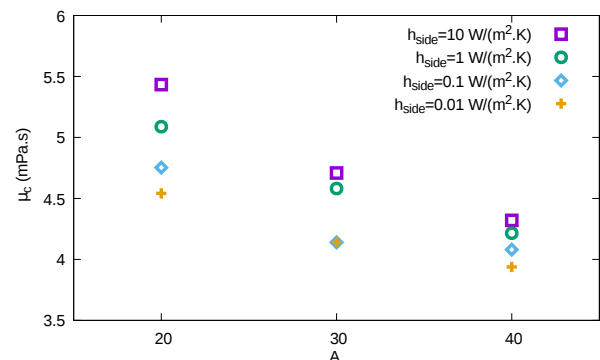

(a) $e=1 \mathrm{~mm}$

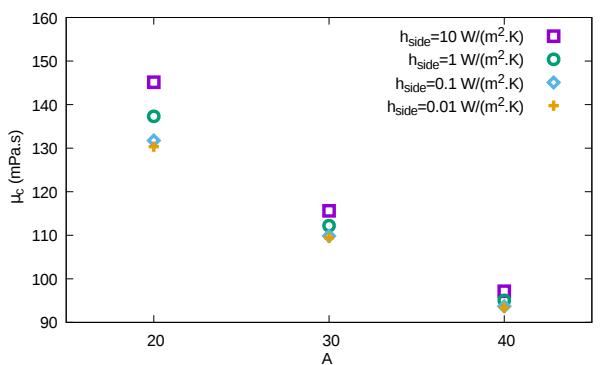

(b) $e=8 \mathrm{~mm}$

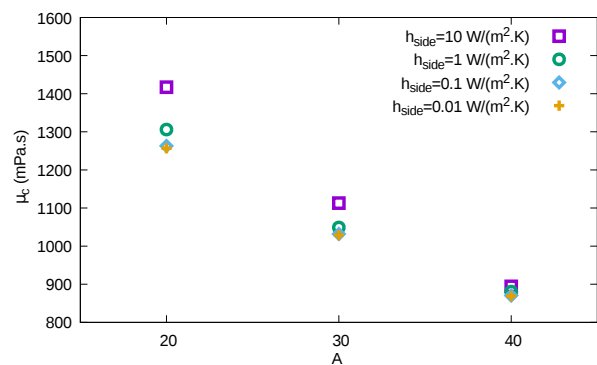

(c) $e=20 \mathrm{~mm}$

Figure 4: Transition thresholds $\mu_{\mathrm{c}}$ as a function of the aspect ratio $A$ for different thicknesses and lateral convective heat transfer coefficients, $r=0$.

Different convective heat transfer coefficients $h_{\text {side }}$ at the lateral walls are investigated. Whatever the $h_{\text {side }}$ and $e$ values, the critical viscosity is clearly a decreasing function of $A$. This behaviour is explained as follows. Let us assume that the viscosity is set to its critical value $\mu_{\mathrm{c}}$. In that case, the convection produced at the lateral walls propagates towards the tested fluid region $(x-A / 2, y) \in[-1,1] \times[0,1]$ so that the core Péclet number grows with time, reaches its peak when $\mathrm{Pe}_{\text {core }}\left(t_{\mathrm{c}}\right)=1$, just before decreasing. If the lateral walls are now moved away from each other, the convection won't be able to reach the core flow layer anymore and the solution will then be considered as diffusive since $\mathrm{Pe}_{\text {core }}(t)<1$ whatever $t$. To recover significant velocities in the central region, the convection must be strengthened by reducing the intensity of the stabilizing contributions, namely increasing the Marangoni and Rayleigh numbers or similarly by reducing the fluid viscosity as shown in Fig. 4. The viscosity decrease seems more pronounced for thick fluid layers: for example between $A=20$ and 30 and for $h_{\text {side }}=10 \mathrm{~W} /\left(\mathrm{m}^{2} \cdot \mathrm{K}\right)$ (violet empty squares), the viscosity is reduced of about $13 \%, 20 \%$ and $21 \%$ for $e=1,8$ and $20 \mathrm{~mm}$, respectively. This suggests that the buoyancy driven flows (moderate and large thicknesses) are intrinsically more stable than capillary driven ones (small thicknesses). 
The fall in the viscosity threshold with the decrease of the lateral convective heat transfer coefficient is also observed in Fig. 4. Indeed, the $h_{\text {side }}$ decrease reduces the intensity of the induced lateral fluid flow. To recover the convection in the core fluid layer, the viscosity that acts to damp out the fluid flow must be diminished. On the whole, three order of magnitude decrease in the convective heat transfer coefficient involves a $\mu_{\mathrm{c}}$ drop of about $10 \%$, whatever the thickness and the aspect ratio, except for $e=1 \mathrm{~mm}$ and $A=20$ where it reaches $\sim 20 \%$. Noticed that if $h_{\text {side }}=0$, the vertical surfaces are adiabatic and no physical perturbation can arise. As discussed in Sec. 3.1, this case is critical since the evolution of the solution would then be controlled mainly by the numerical errors (solver, accuracy, ...), what would have no physical relevance.

\subsection{Spatio-temporal diagrams: physical versus numerical disturbances}

Figure 5(a) presents the spatio-temporal diagram obtained by reporting the fluid velocity at the free surface $U(x, y=1 ; t)$ as a function of time, for $e=1 \mathrm{~mm}, h_{\text {side }}=10 \mathrm{~W} /\left(\mathrm{m}^{2} \cdot \mathrm{K}\right), A=20$ and a viscosity $40 \%$ smaller than $\mu_{\mathrm{c}}$ (see Fig. $4(\mathrm{a})$ ). Snapshots of this flow field are drawn in Fig. 3. Figures 3 and 5(a) clearly show that the onset of the convection starts near

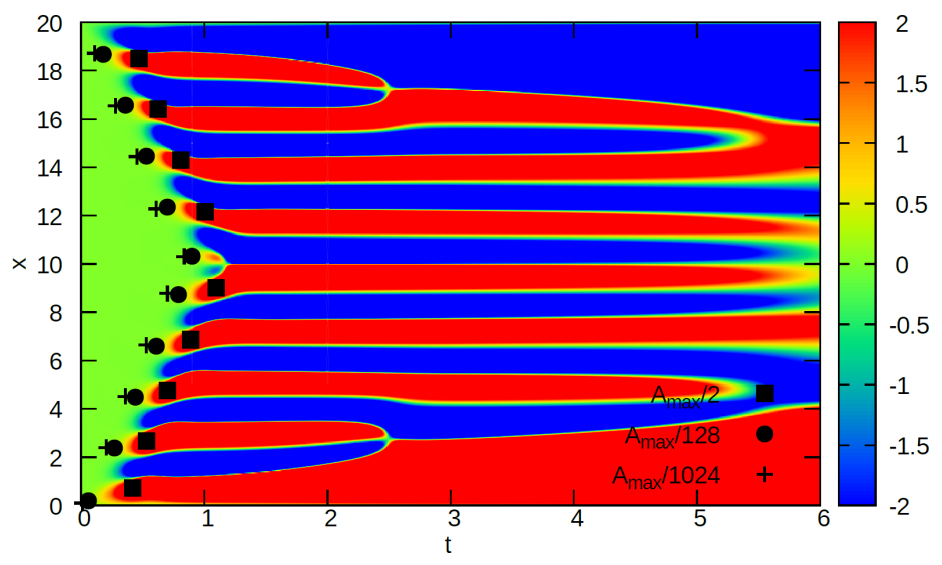

(a) $A=20$

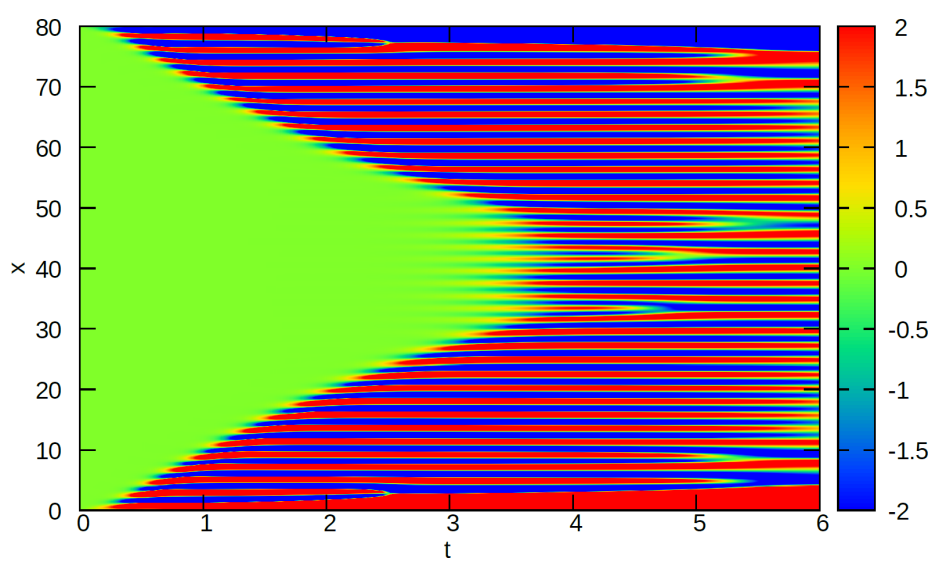

(b) $A=80$

Figure 5: Horizontal velocity at the free surface as a function of time, for $e=1 \mathrm{~mm}, \mu=3.25 \mathrm{mPa} \cdot \mathrm{s}, h_{\text {side }}=10 \mathrm{~W} /\left(\mathrm{m}^{2} \cdot \mathrm{K}\right)$ and $r=0$. (a) Small extension $A=20$. The symbols stand for the loci where the maximal velocity of each convective cell reaches the magnitude $A_{\max } / 2^{n}(n=1,7$ and 10$)$ with $A_{\max }=\max _{x, t}[U(x, y=1 ; t)]$ the maximal velocity. The stream lines at times $t=0.5,1,1.5,3$ and 6 are drawn in Fig. 3. (b) Large extension $A=80$.

the vertical walls before spreading over the whole fluid domain. This gives rise to the occurrence of new circulation cells which are rotating in alternated directions, as illustrated by the red and blue stripes in the spatio-temporal diagram. These convective cells evolve in number, size and shape while keeping the symmetry around $x=A / 2=10$. The reorganization of the convective cells at $t \approx 2.5$ and 5.5 is robust since it is also observed for the larger extension $A=80$ (see Fig. 5 (b) close to the boundaries $x=0$ and 80$)$.

For large aspect ratios, Fig. 5 (b) for $A=80$, in addition to the propagation of the convection that occurs gradually from the boundaries to the core fluid layer, convective cells appear suddenly at the centre of the domain for $t \sim 3.5$. The specific behaviour of this latter spatio-temporal diagram is due to the existence of two time scales that trigger the convection onset. One has a physical origin and is due to the flow induced by the convective heat transfer along the lateral walls. The other results from the growth of a local disturbance. For large aspect ratios, the second mode of destabilization seems to prevail in the core fluid layer. To explain its origin, extra simulations are performed with the same parameters $\left(e=1 \mathrm{~mm}, \mu=3.25 \mathrm{mPa} \cdot \mathrm{s}, h_{\text {side }}=10 \mathrm{~W} /\left(\mathrm{m}^{2} \cdot \mathrm{K}\right)\right.$ and $\left.A=80\right)$, and by adding different small disturbances of maximal magnitude $r$, on the initial temperature field (see Eq. (3)). Figure 6(a), showing the spatio-temporal diagrams achieved with $r=10^{-6}, 10^{-9}$ and $10^{-12}$, clearly points out that the strengthening of the initial disturbance brings forward the onset of the destabilization in the core fluid layer. These artificial initial perturbations act roughly as the spurious perturbations stemming from the numerical solving (Navier-Stokes solver, number of significant digits, ...). To confirm this assertion, two additional simulations are carried out with the same main parameters, but without any heat transfer at the lateral wall $\left(h_{\text {side }}=0\right)$ and any initial perturbation $(r=0)$, but with two different algorithms to uncouple the pressure and velocity variables. The first simulation is performed with the current finite volume code that uses the incremental pressure correction scheme [9]. The second one is based on a pseudo-spectral code with a velocity correction method [2, 11]. The comparison of the two spatio-temporal diagrams in Fig. 6(b) clearly shows that the numerical choices to solve the Navier-Stokes equations play an important role on the convection onset when the solution is let free to evolve without any control of the nature of the perturbation, neither at the lateral walls $\left(h_{\text {side }}=0\right)$ nor at the initial time $(r=0)$. With the pressure correction method (lower picture in Fig. 6(b)), the convective cells emerge close to the wall like it was obtained for a non zero convective heat transfer coefficient, but more belatedly; afterwards, they are propagated towards the core fluid layer. This destabilization is related to the appearance of spurious numerical boundary layers along the walls which are due to the pressure-velocity 


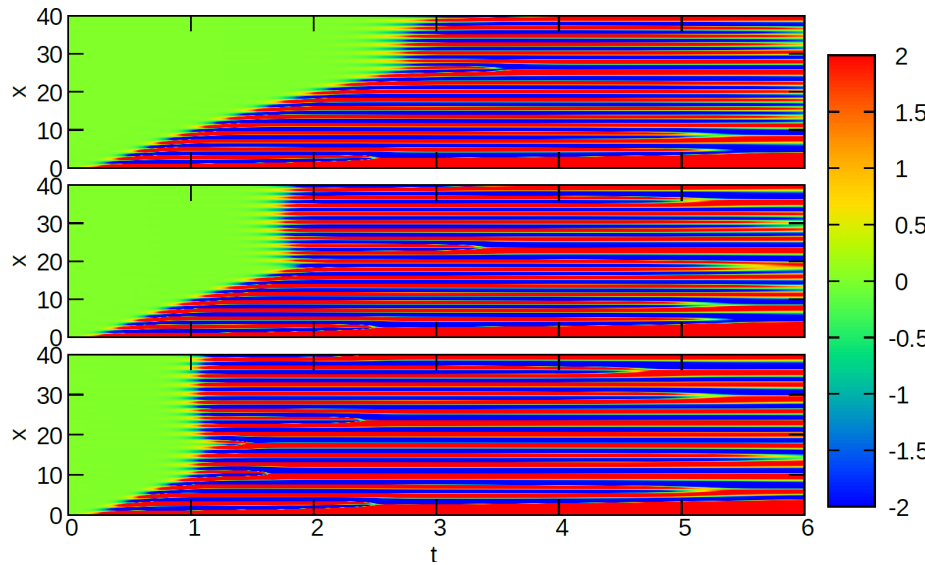

(a) From bottom to top: $r=10^{-6}, 10^{-9}$ and $10^{-12}$.

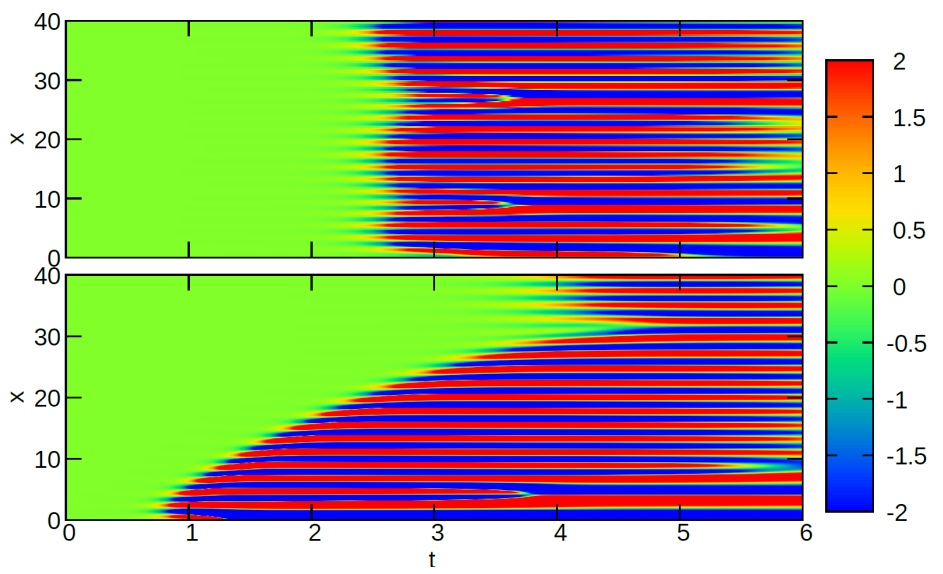

(b) Bottom: Finite volume with a incremental pressure correction scheme; top: spectral method with a velocity correction scheme.

Figure 6: Horizontal velocity at the free surface as a function of time for $e=1 \mathrm{~mm}, \mu=3.25 \mathrm{mPa} \cdot \mathrm{s}$ and $A=80$. (a) $h_{\text {side }}=10 \mathrm{~W} /\left(\mathrm{m}^{2} \cdot \mathrm{K}\right)$ with different initial temperature perturbations of magnitude $r$. (b) $h_{\text {side }}=0$ and $r=0$ for two incompressible Navier-Stokes solvers.

decoupling of the pressure correction method [10]. The velocity correction method [2, 11] does not suffer such a drawback. However in this latter case, since the continuity equation is not perfectly satisfied in the bulk fluid, the instability arises simultaneously everywhere in the fluid domain (upper picture in Fig. 6(b)). From the analysis of these two last simulations, it could then be argued that the projection-like methods are unadapted to handle such physical problem, and an "exact" solver for the Navier-Stokes equations should be used. Although this remark seems a-priori pertinent, such solvers cannot become independent from the other numerical errors (number of significant digits, accuracy of the linear solvers, ill-conditioned matrices, ...). To conclude this discussion, it is then necessary to free from any spurious numerical destabilization to ensure a physical and predictable behaviour of the numerical solution. This is achieved by controlling explicitly the destabilization source. In this work, it simply amounts to considering a weak heat transfer at the lateral walls. However for $A=80$ (Fig 5(b)), despite the lateral disturbances, the core fluid remains controlled by the numerical perturbations. And it is the reason why the critical viscosities $\mu_{\mathrm{c}}$ obtained for $A>40$ are in fact not presented in Fig. 4.

\subsection{Propagation celerity of the convection wave}

To characterize the propagation phenomena, the maximal velocity magnitude at the free surface of each circulation cells has been recorded with its abscissa, as a function of time. From these temporal data, we have extracted the times and the corresponding abscissas for which the velocity magnitudes are equal to the prescribed values $\pm A_{\max } / 2^{n}, n=1, \ldots, 10$, with $A_{\max }=\max _{x, t}[U(x, y=1 ; t)]$. In this algorithm, only $(x, t)$-coordinates associated with cells that were originated from the lateral walls were accounted for. An example of such coordinates is plotted on Fig. 5(a) for $n=1,7$ and 10 and positive velocities only. After having made use of the symmetry of the diagram around the mid-plane $x=A / 2$, the set of points obtained for a fixed $n$ value is nearly aligned according to a straight line. The propagation celerity of the convection wave is then evaluated by a linear regression. Figure 7 gives this celerity $c_{n=1,10}$ as a function of the aspect ratio $A$, for $e=1,8$ and

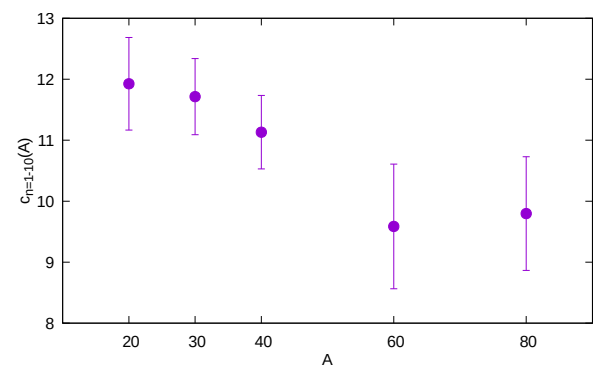

(a) $e=1 \mathrm{~mm}, \mu=3.25 \mathrm{mPa} \cdot \mathrm{s}$

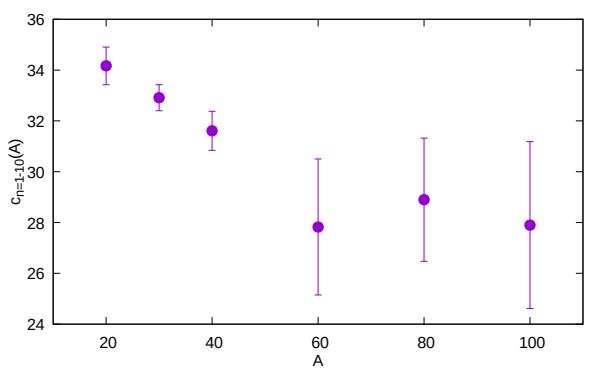

(b) $e=8 \mathrm{~mm}, \mu=70 \mathrm{mPa} \cdot \mathrm{s}$

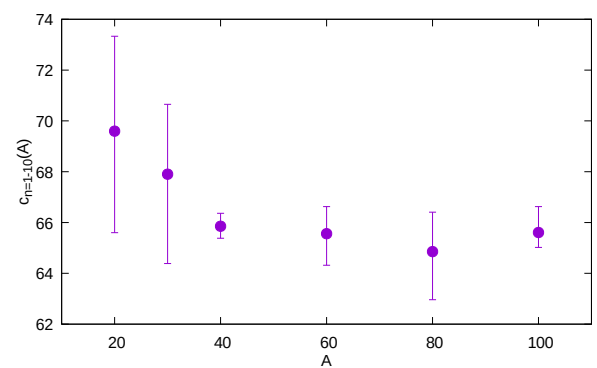

(c) $e=20 \mathrm{~mm}, \mu=500 \mathrm{mPa} \cdot \mathrm{s}$

Figure 7: Propagation celerity of the convection wave as a function of the aspect ratio. The vertical bars take into account the different amplitudes $A_{\max } / 2^{n}, n=1$ to 10 , and the filled circle stand for the mean value. Parameters: $h_{\text {side }}=10$ $\mathrm{W} /\left(\mathrm{m}^{2} \cdot \mathrm{K}\right)$ and $r=0$.

$20 \mathrm{~mm}$. The scattering of the celerity for a given aspect ratio $A$ is at most about $\pm 15 \%$ of the average value given by the 
filled circles. For each thickness, two different behaviours can be identified depending on whether the aspect ratio is small or large. This partition takes place for $A \simeq 60$ when $e=1$ and $8 \mathrm{~mm}$, and $A \simeq 40$ when $e=20 \mathrm{~mm}$. For large wall-to-wall spacings, the mean wave celerity is rather constant, independent of the aspect ratio. If the aspect ratio decreases enough, the waves seem to propagate rather faster, indicating that the confinement effects promote the development of the convective cells.

The sensitivity of the mean propagation celerity of the convection wave is analysed via a parametric study in the (Ma,Ra) plane for $A=20$ and $\mathrm{Bi}_{\text {side }}=0.07$ ( $h_{\text {side }}$ variable). Figure 8 , which provides the celerity map as a function of the Rayleigh

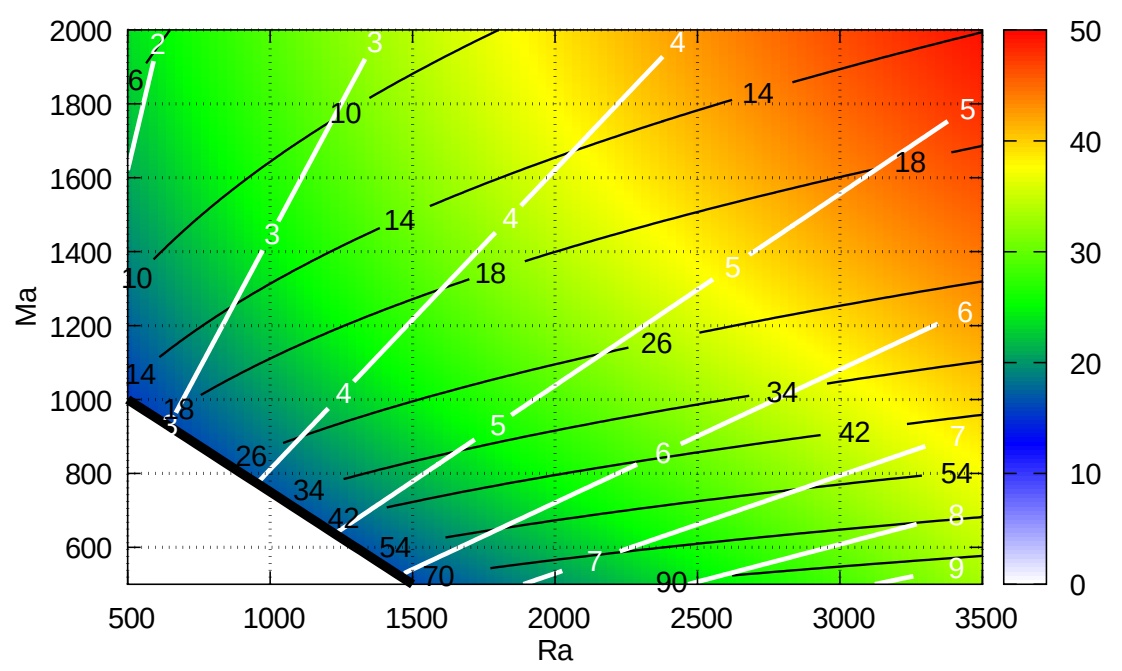

Figure 8: Mean propagation celerity of the convection wave as a function of Ma and Ra. Iso thicknesses in [mm] (white lines) and viscosities in $[\mathrm{mPa} \cdot \mathrm{s}]$ (black lines) are also represented. The uncoloured region corresponds to transient diffusive solutions in the core domain $\left(\mathrm{Pe}_{\text {core }}(t)<1, \forall t>0\right)$. Parameters: $\mathrm{Bi}_{\text {side }}=0.07, A=20$ and $r=0$.

and Marangoni numbers, indicates that the wave celerity increases with $\mathrm{Ma}$ and $\mathrm{Ra}$, or with the physical parameters $1 / \mu$

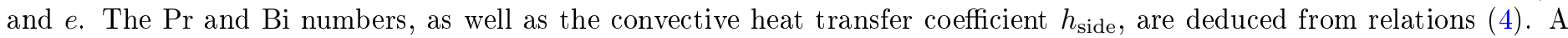
bi-quadratic analytical approximation $\tilde{c}$ of the numerical celerity has been formulated by means of the least-square method:

$$
\tilde{c}(\mathrm{Ma}, \mathrm{Ra})=\sum_{i=0}^{2} \sum_{j=0}^{2} a_{i, j} \mathrm{Ra}^{i} \mathrm{Ma}^{j}
$$

with the coefficients $a_{0,0}=2.49, a_{1,0}=-0.002, a_{0,1}=0.008, a_{1,1}=1.73 \times 10^{-5}, a_{2,0}=2.38 \times 10^{-6}, a_{0,2}=-3 \times 10^{-7}$, $a_{1,2}=-4.95 \times 10^{-9}, a_{2,1}=-4.13 \times 10^{-9}$ and $a_{2,2}=1.21 \times 10^{-12}$. The relative differences between the analytical and numerical celerity $|\tilde{c} / c-1|$ (indicated in brackets in Tab. 1) indicates that the approximation (8) is accurate. Notice that the

\begin{tabular}{c||cccc} 
Marangoni $\downarrow$ Rayleigh $\rightarrow$ & 500 & 1500 & 2500 & 3500 \\
\hline 500 & - & $16.06(0.88 \%)$ & $24.20(1.64 \%)$ & $33.43(1.52 \%)$ \\
1000 & $15.17(0.37 \%)$ & $24.89(1.56 \%)$ & $33.00(0.93 \%)$ & $40.58(1.91 \%)$ \\
1500 & $29.63(1.60 \%)$ & $31.10(1.69 \%)$ & $38.79(0.58 \%)$ & $45.90(1.35 \%)$ \\
2000 & $23.06(1.62 \%)$ & $34.88(2.10 \%)$ & $42.56(0.54 \%)$ & $50.29(1.87 \%)$
\end{tabular}

Table 1: Mean propagation celerity $c$ of the convection wave. The bracketed values are the relative differences between the simulation and the correlation function $(8),|\tilde{c} / c-1|$. The solution at $\mathrm{Ra}=500$ and $\mathrm{Ma}=500$ is diffusive: no convective cell reaches the core fluid layer $\left(\mathrm{Pe}_{\text {core }}(t)<1, \forall t>0\right)$. Parameters: $\mathrm{Bi}_{\text {side }}=0.07, A=20$ and $r=0$.

variation range of the celerity is large enough to neglect the scattering produced by the different tested values $\pm A_{\text {max }} / 2^{n}$, from $n=1$ to 10 .

\subsection{Critical time for the convection onset}

The critical times $t_{\mathrm{c}}$ (Eq. (7)) for the onset of the convection in the core fluid layer are given in Fig. 9 for the threshold viscosities $\mu_{\mathrm{c}}$ and three critical thicknesses. The analysis of $t_{\mathrm{c}}$ as a function of the aspect ratio $A$ is delicate because of different antagonistic effects. On the one hand, the time $t_{c}$ is expected to increase when the vertical walls are moved apart because (a) the convection wave has to cover a longer distance to propagate from the lateral boundaries toward the core fluid layer, and (b) the celerity of this wave tends to decrease as a function of $A$ (see Fig. 7 for $A \leq 40$ ), if the viscosity is kept constant. On the other hand from Fig. 4, the increases in the aspect ratio leads to a decrease in the critical viscosity. 


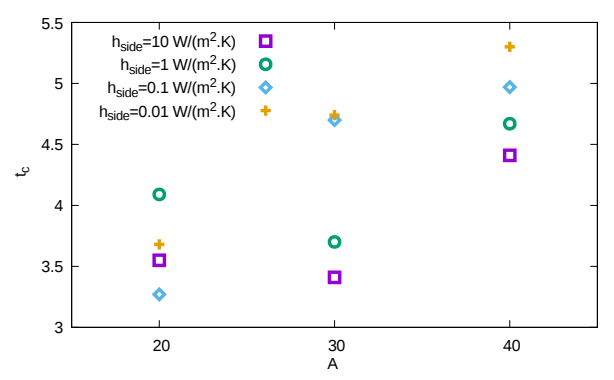

(a) $e=1 \mathrm{~mm}$

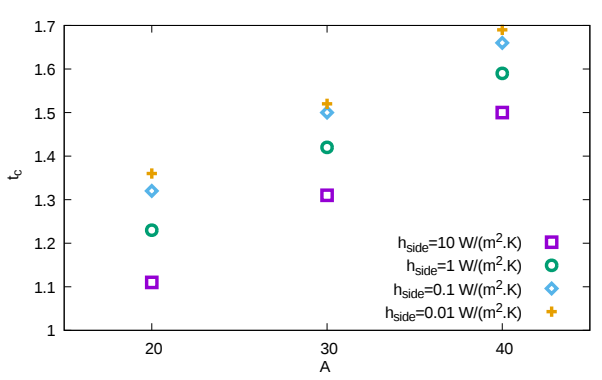

(b) $e=8 \mathrm{~mm}$

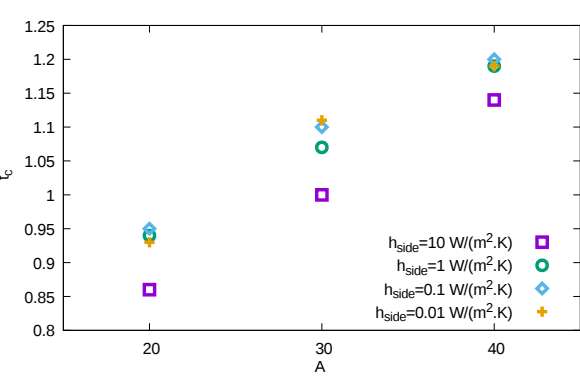

(c) $e=20 \mathrm{~mm}$

Figure 9: Transition times $t_{\mathrm{c}}$ as a function of the aspect ratio $A$ for different thicknesses and lateral convective heat transfer coefficients, $r=0$.

Let us assume that the validity range of the analytical celerity expression $\tilde{c}(\mathrm{Ra}, \mathrm{Ma})$ (Eq. (8)), obtained with a fixed aspect ratio, can be prolonged for $\mathrm{Ra}$ and Ma parameters close to their critical values. Then, reducing the viscosity (successive black isolines with same white isoline in Fig. 8) gives rise to a growth of the wave celerity $c$, denoted $\delta c>0$. Considering $t_{\mathrm{c}}$, the time required for the wave to reach the cavity centre, we approximatively have $c \times t_{\mathrm{c}} \simeq A / 2$. A small variation in the aspect ratio $\delta A$ leads to a variation $\delta t_{\mathrm{c}}$ of $t_{\mathrm{c}}$, in order of $\delta t_{\mathrm{c}}=\delta A /(2 c)-t_{\mathrm{c}} \times \delta c / c$. Therefore, the critical time $t_{\mathrm{c}}$ for the convection to travel from the walls to the core fluid layer will be reduced $\left(\delta t_{\mathrm{c}}<0\right)$, if $\delta A / 2<t_{\mathrm{c}} \times \delta c$. From Fig. 9 , $t_{\mathrm{c}}$ seems overall rather to rise with the aspect ratio. This would suggest that the celerity increase due to the decrease in the critical viscosity is insufficient to counterbalance the increase in the length between the central fluid layer and the lateral walls.

As mentioned in Sec. 3.3, the decrease in the convective heat transfer coefficient $h_{\text {side }}$ leads to a reduction of the critical viscosity, when $A$ and $e$ are kept constant (Fig. 4). As previously, if we assume that the approximated celerity $\tilde{c}($ Ra, Ma) (Eq. 8) is valid for parameters close to their critical values, the threshold time $t_{\mathrm{c}}$ would be reduced when $h_{\text {side }}$ is decreased. However, this analysis seems rather inconsistent with the numerical results shown in Fig. 9. This apparent contradiction may be explained by looking more carefully at the spatio-temporal diagrams for different convective heat transfer coefficients, for example for $e=8 \mathrm{~mm}$ and $A=20$ (Fig. 10). The decrease in $h_{\text {side }}$ reduces the heat transfer through the vertical walls and

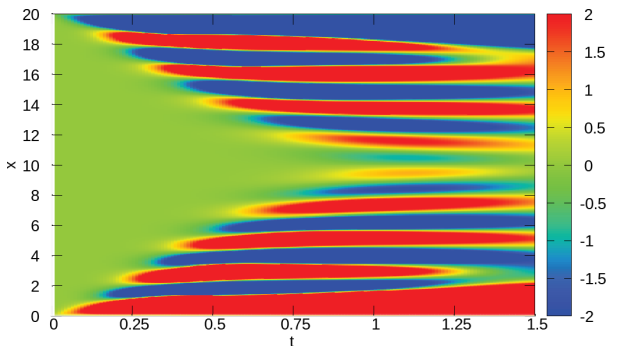

(a) $h_{\text {side }}=10 \mathrm{~W} /\left(\mathrm{m}^{2} \cdot \mathrm{K}\right), \mu=145 \mathrm{mPa} \cdot \mathrm{s}$

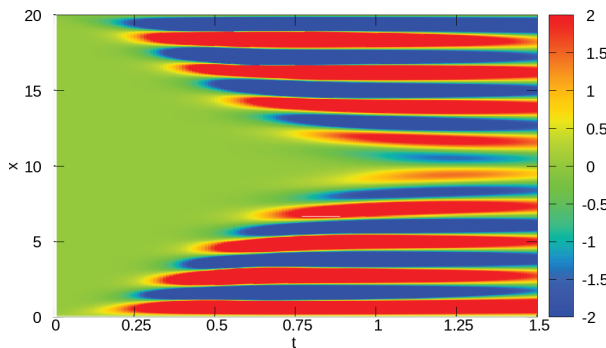

(b) $h_{\text {side }}=1 \mathrm{~W} /\left(\mathrm{m}^{2} \cdot \mathrm{K}\right), \mu=135 \mathrm{mPa} \cdot \mathrm{s}$

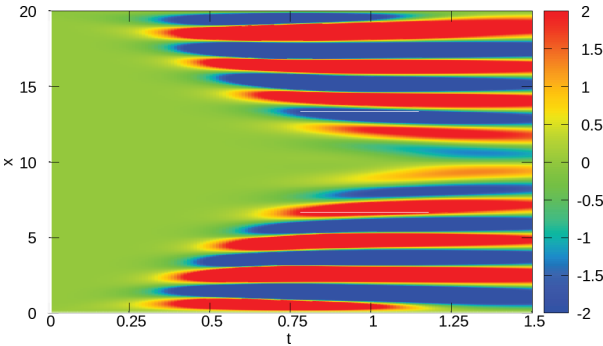

(c) $h_{\text {side }}=0.1 \mathrm{~W} /\left(\mathrm{m}^{2} \cdot \mathrm{K}\right), \mu=130 \mathrm{mPa} \cdot \mathrm{s}$

Figure 10: Horizontal velocity at the free surface as a function of time, for $e=8 \mathrm{~mm}, \mu \lesssim \mu_{\mathrm{c}}$ and $r=0$.

therefore the intensity of the local convective flow. But the main feature concerns the delayed onset of the convection close to the walls. This is due to the temporal variation of diffusive temperature field which decreases from $\Theta=0\left(T=T_{0}\right)$ to $\Theta=-1\left(T=T_{0}-L \Phi_{s} / H\right)$. Since $h_{\text {side }}$ decreases, the required time $t$ to get a significant heat flux $\left(h_{\text {side }}\left(T(t)-T_{0}\right)\right)$ becomes longer: the appearance of a convection seed along the lateral walls is consequently delayed.

\subsection{Convection cell characterization}

The characterization of the convective cells is based on spatio-temporal diagrams which provide the velocity at the free surface as a function of time. The adopted procedure is as follows. For each time, the different abscissa where the horizontal velocity cancels are recorded. If the Péclet number reaches or exceeds the unity between two adjacent zero positions, the so defined interval is then identified as the signature of a convective pattern; otherwise the local region is assumed diffusive. To avoid bias in the analysis, the two large convection cells adjacent to the lateral walls are disregarded. Figure 11 shows the evolution of the number of convection cells $N_{\text {cell }}$ for $h_{\text {side }}=10 \mathrm{~W} /\left(\mathrm{m}^{2} \cdot \mathrm{K}\right), e=1,8$ and 20 mm with viscosities respectively about $40 \%, 50 \%$ and $60 \%$ smaller than the critical ones and a large range of aspect ratios $A$. The solution is firstly diffusive $\left(N_{\text {cell }}=0\right)$ for short times, then convective cells become visible and their numbers increase. This augmentation is not sensitive to the aspect ratio $A$; only the plateau corresponding to the maximum number of cells differs. For large aspect ratios, a sudden increase in $N_{\text {cell }}$ is observed at $t=3.5,1$, and 0.4 for $e=1,8$ and $20 \mathrm{~mm}$, respectively. As already shown in 


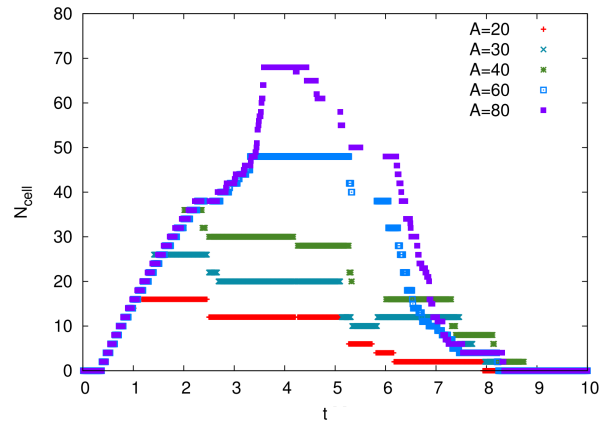

(a) $e=1 \mathrm{~mm}, \mu=3.25 \mathrm{mPa} \cdot \mathrm{s}$

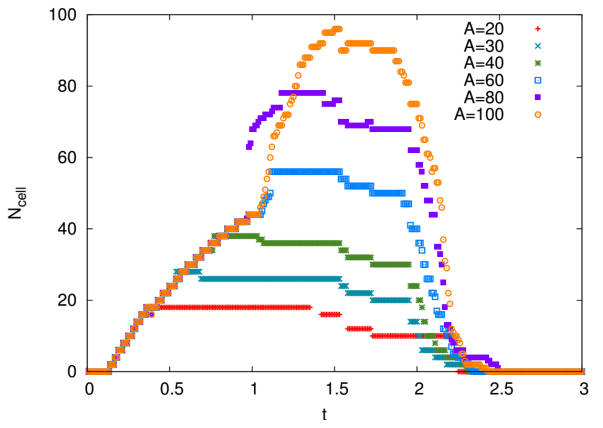

(b) $e=8 \mathrm{~mm}, \mu=70 \mathrm{mPa} \cdot \mathrm{s}$

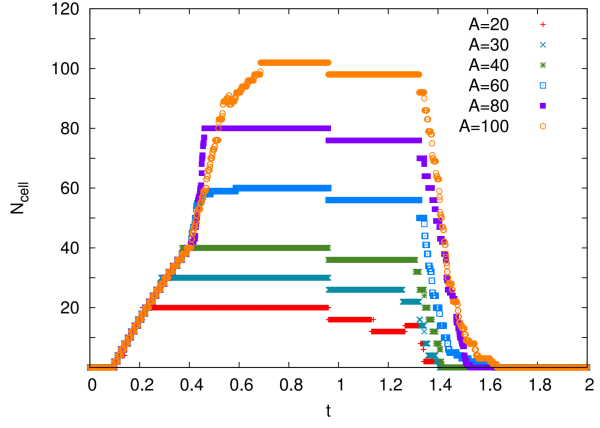

(c) $e=20 \mathrm{~mm}, \mu=500 \mathrm{mPa} \cdot \mathrm{s}$

Figure 11: Evolution of the number of convective cells $N_{\text {cell }}$ for different aspect ratios, $h_{\text {side }}=10 \mathrm{~W} /\left(\mathrm{m}^{2} \cdot \mathrm{K}\right)$ and $r=0$.

Fig. 5(b), this behaviour is due to numerical perturbations whose growth rates are greater than those of the convective cells propagating from the wall to the core flow region. Once the whole fluid domain is filled by convective cells, their number remains nearly constant or slightly decreases because of local rearrangements (see for example Fig. 11(a) for $A=20$ and at $t \sim 2.5$, and the diagram 5(a) at the same time). Finally for longer times, the number of convective cells weakens smoothly before the fluid solution becomes again at rest, except at the wall vicinity where the convection remains intense (uncounted cells). It is worth noticing that the moment from which the decrease in the cell number is significant is almost independent of the aspect ratio $A$, in the order of $t \sim 5,2$ and 1.3 for $e=1,8$ and $20 \mathrm{~mm}$. For $e=1 \mathrm{~mm}$, the interval of the cell decrease is more extended than for the two other thicknesses.

Figure 12 gives the averaged cell size $\lambda_{\text {av }}$ at some discrete times, for various thicknesses and aspect ratios. This average

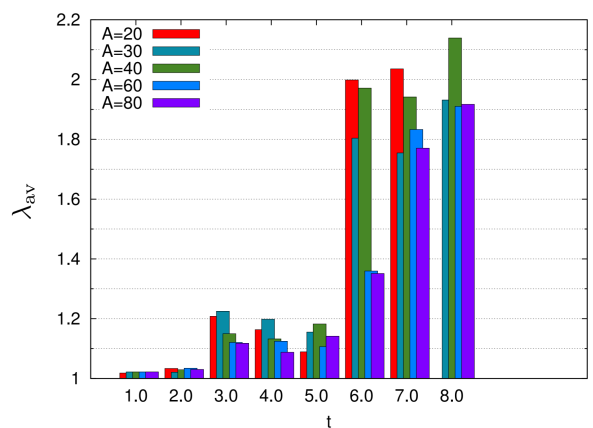

(a) $e=1 \mathrm{~mm}, \mu=3.25 \mathrm{mPa} \cdot \mathrm{s}, \Delta \tau=1$

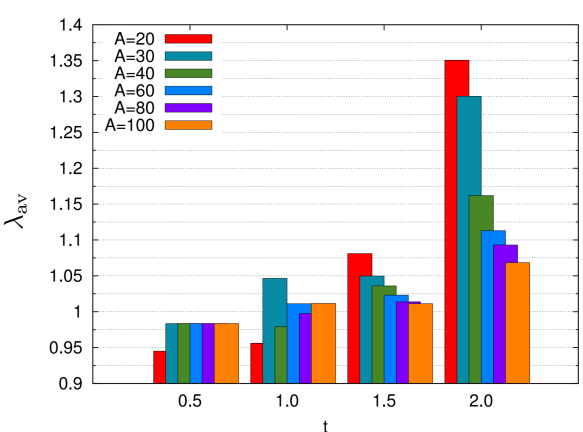

(b) $e=8 \mathrm{~mm}, \mu=70 \mathrm{mPa} \cdot \mathrm{s}, \Delta \tau=0.5$

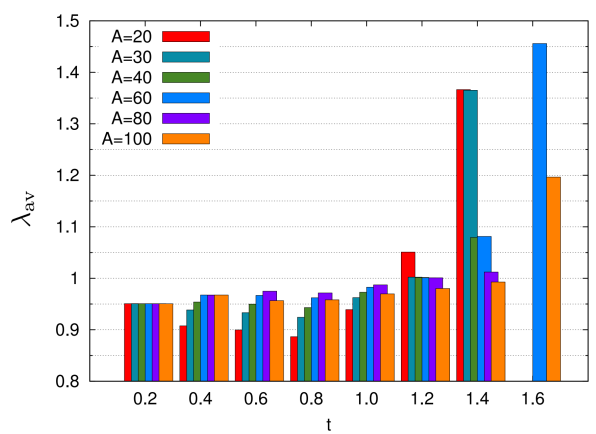

(c) $e=20 \mathrm{~mm}, \mu=500 \mathrm{mPa} \cdot \mathrm{s}, \Delta \tau=0.2$

Figure 12: Evolution of the average size of the convective cells $\lambda_{\mathrm{av}}$ for different aspect ratios, $h_{\mathrm{side}}=10 \mathrm{~W} /\left(\mathrm{m}^{2} \cdot \mathrm{K}\right)$ and $r=0$.

is carried out over the whole convective cells and time intervals $\Delta \tau$ which are indicated in the captions. For the three thicknesses, the averaged cell size is nearly constant $\left(\lambda_{\mathrm{av}}=1 \pm 10 \%\right)$, both in the propagation and plateau regimes, as long as the number of convective cells does not fall sharply. This numerical value indicates that, as usually, the length scale for the convection cells are related to the fluid thickness. A slight difference is noticed for the smallest thickness and $3 \lesssim t \lesssim 5$ where the average cell size is a bit greater, in the order of $1.15 \pm 5 \%$. When the fluid flow locally relaxes towards the diffusive state, the remaining convective cells get significantly longer, mainly for $e=1 \mathrm{~mm}$ (see Fig. 12(a) and the snapshot 3(e) for $A=20$ and $t=6)$.

\subsection{Perturbations induced at the initial time}

In the former works by Touazi et al. [18] and Trouette et al. [20, 21], the convection seed was originated from the initial conditions by adding small random disturbances, either on the velocity or the temperature field. To this end, we proceed the same way by suppressing the heat transfer at the lateral wall $\left(B_{i_{\text {side }}}=0\right.$ in Eq. (2a)) and introducing a spatial perturbation on the temperature field, chosen in a uniform distribution with a magnitude $r=10^{-3}$ (see Eq. (3)).

Figure 13(a) shows the spatio-temporal diagram for the horizontal velocity at the free surface for $e=1 \mathrm{~mm}, \mu=3.25$ $\mathrm{mPa} \cdot \mathrm{s}$ and $A=20$. Introducing disturbances at the initial instant modifies substantially the convection at the free surface (see Fig. 5(a) for comparison), namely the convection onset and the evolutions of both the cell number (Fig. 13(b) versus Fig. 11(a)) and the cell size (Fig. 13(c) versus Fig. 12(a)). The simultaneous appearance of the convective cells in the whole 


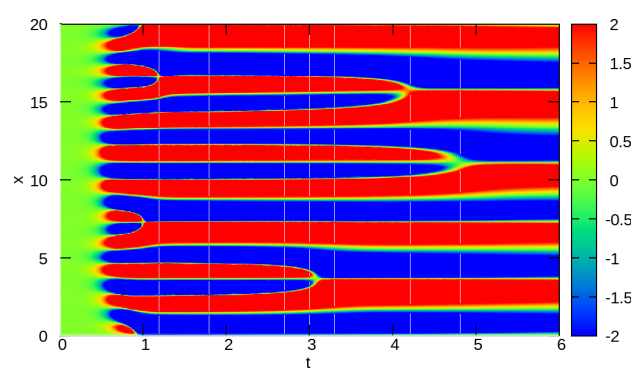

(a) Spatio temporal diagram

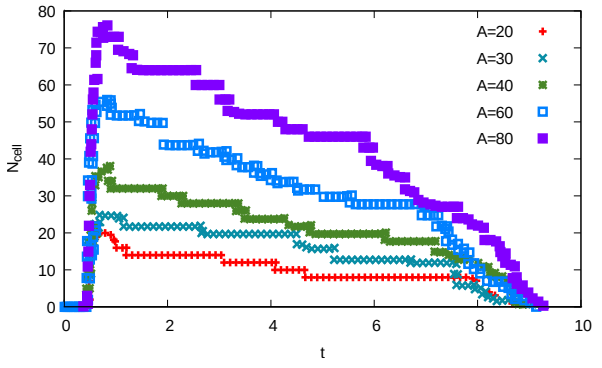

(b) Evolution of the number of convective cells

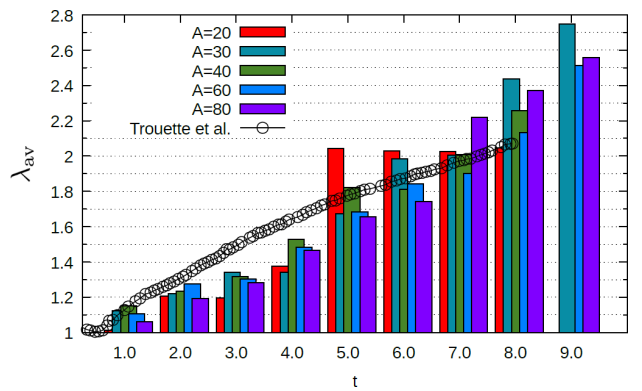

(c) $\lambda_{\text {av }}$ Evolution of the average convective cell size at the discrete times $t \in \mathcal{N}[1,9]$ (average over $\Delta \tau=1)$ and data by Trouette et al. [20] $(\mu=3 \mathrm{mPa} \cdot \mathrm{s}$ and $A=20)$

Figure 13: Evolution of the solution for $e=1 \mathrm{~mm}, \mu=3.25 \mathrm{mPa} \cdot \mathrm{s}, h_{\text {side }}=0$ and $r=10^{-3}$.

fluid layer is a common characteristic of the evolution of the fluid flow when the perturbations are set at $t=0$. Therefore, the time corresponding to the convection onset is nearly insensitive to the aspect ratio $A$ (Fig. 13(b)). The shape of the random disturbance does nearly not influence it also, contrary to the intensity $r$ that moves it forward or backward according to whether the $r$-value is larger or smaller (see Fig. 6(a) as an illustrative example). Since the perturbation is chosen randomly, the spatio-temporal diagram does not fulfil the symmetry condition about the mid plane $x=A / 2$, as it was when a convective heat transfer was imposed at the lateral walls. Different initial conditions lead to slightly different diagrams, but the overall evolution of the number and size of the convective cells are qualitatively the same. For the thin liquid film $e=1 \mathrm{~mm}$, the increase in the cell size with time looks smoother than in the case where the heat transfer at the vertical walls are activated (see Figs. 12(a) and 13(c)). Notice that this difference is much less pronounced for the thicker fluid layers, $e=8$ and 20 mm [3]. In Fig. 13(c) is also drawn the evolution of the average cell size obtained by Trouette et al. [20], also for $e=1 \mathrm{~mm}$ and $A=20$, but with $\mu=3 \mathrm{mPa} \cdot \mathrm{s}$. The slow and regular increase in the $N_{\text {cell }}$ is qualitatively recovered. Quantitative comparisons are difficult because the viscosity, the way the cell size is determined, and the initial temperature perturbation differ.

\section{Summary and concluding remarks}

In this paper, the transition between a diffusive and convective flow regime, induced by the evaporation of a Polyisobutylene/Toluene solution, is numerically studied. Since the flow evolution is transient, it is necessary to control the nature of the disturbance that will give rise to convection; otherwise the destabilization won't be physical, but numerical in nature. Contrary to the approach used in previous works, where random perturbations are imposed at the initial condition. We have adopted here a model in which the disturbance is induced at the lateral walls, through a heat transfer. To proceed to the evaluation of the transition between an essentially diffusive solution to a convective flow, we have used a criteria based on the Péclet number evaluated in the core fluid layer, far away from the lateral boundaries. The critical viscosity has been determined for different aspect ratios of the fluid layer, three thicknesses and a large range of heat transfer coefficients. The investigation of the convection flow has been done by means of spatio-temporal diagrams representing the free surface velocity as a function of time. This has enabled us to study the celerity of the convection wave sourced from the lateral walls, the evolution of the convection cells, both in size and number.

Finally, the sensitivity of the convection onset to the way the solution is destabilized is illustrated in Fig. 14 which sums up the critical viscosities $\mu_{\mathrm{c}}$ obtained in this study, and the thresholds given by Touazi et al. (personal data and [18]), but for disturbances applied on the velocity field instead of on the temperature field. This figure clearly emphasizes that the thresholds depend on the adopted choices to perturb the solution, either at the initial condition, or at the boundary, but also the criteria to decide whether the flow is convective or not. The main advantage of our approach, which consists in accounting the heat transfer at the lateral walls, is that the solution is determinist and easily reproducible (same threshold and spatio-temporal diagram for example).

Some comparisons may also be performed with the experimental measurements on PIB solutions [19] (Fig. 15). The convective/diffusive nature of flows was achieved through visualizations of the free surface motions with a Marlin digital camera, the solution being seeded with iriodin particles. For few experiments, top views were also performed with an IR camera to provide information on the surface temperature field which is more sensitive to exhibit convection flows. Our transition thresholds between a diffusion state and a convective flow are in good agreement with the $2 \mathrm{D}$ numerical transition line by Touazi et al. [18]. Notice that the violet square symbols in Fig. 14 are identical to this transition line for our three thicknesses of interest. By in large, the simulations seem to underestimate the critical viscosities, what means that the experimental liquid layers are more unstable than the numerical ones. This difference may be explained if we consider some experimental perturbations 

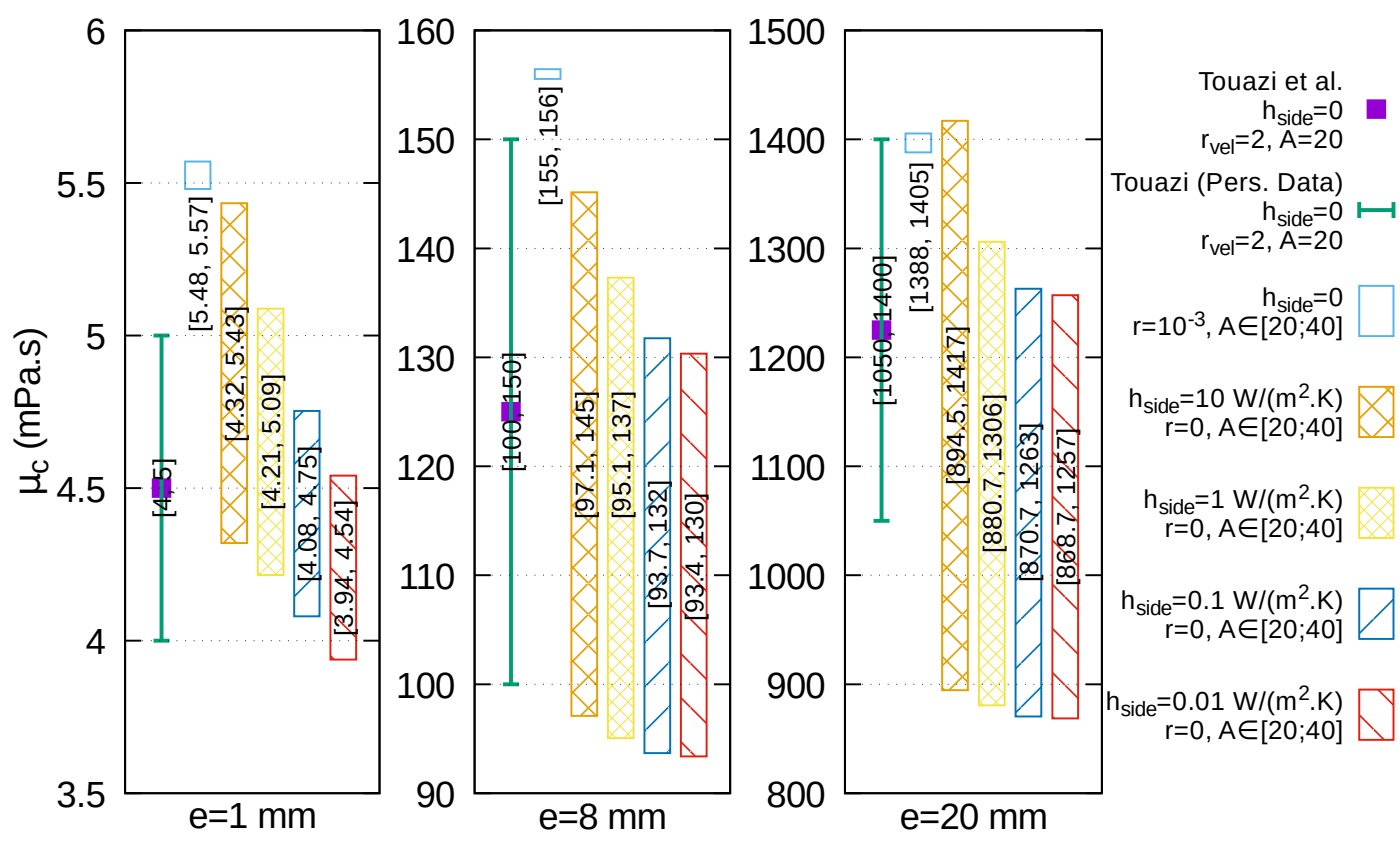

Figure 14: Synthesis of the critical viscosity $\mu_{\mathrm{c}}$ for disturbances produced along the lateral walls $\left(h_{\text {side }}=10 \mathrm{~W} /\left(\mathrm{m}^{2} \cdot \mathrm{K}\right)\right.$ and $r=0)$ and at the initial condition $\left(h_{\text {side }}=0\right.$ and $\left.r=10^{-3}\right)$. Comparison with the Touazi's personal data and by Touazi et al. [18], both obtained for random disturbances of magnitude $r_{\mathrm{vel}}=2$ applied on the velocity field. Each box given in the Touazi's personal data indicates the best obtained framing of the viscosity threshold, on the one hand by a convective solution (lower bound) and on the other hand by a diffusive one (upper bound) (see Fig. 2 for example). Published data in [18] are the average values of the aforementioned data.

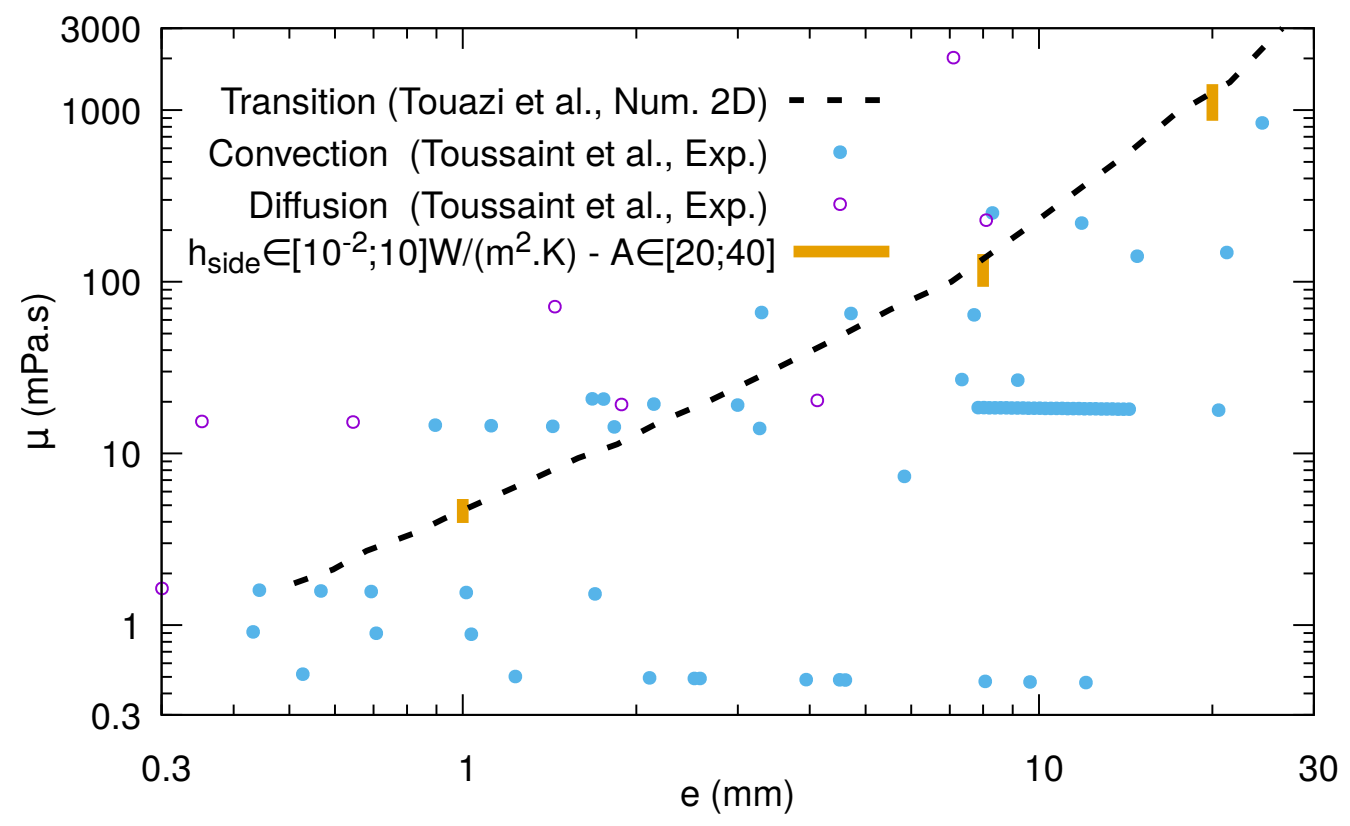

Figure 15: Comparison between the critical viscosity intervals defined for $h_{\text {side }} \in[0.01 ; 10] \mathrm{W} /\left(\mathrm{m}^{2} \cdot \mathrm{K}\right), A \in[20 ; 40], r=0$ and three thicknesses $(e=1,8$ and $20 \mathrm{~mm}$ ), the experimental data by Toussaint et al. [19] and the 2D numerical transition line by Touazi et al. [18].

which promote the convection in the liquid layer. This assumption seems valid if we refer to the experimental set-up where an extracting hood is used to control the solvent concentration far from the solution. The consequence of this sucking up is a non negligible air velocity close the film surface that was evaluated by the experimentalists of about $0.1 \mathrm{~m} / \mathrm{s}$. Accounting for this destabilizing effect, we can reasonably conclude that our numerical results are in qualitative agreement with the experiments. 
This work was granted access to the HPC resources of IDRIS under the allocation 2017-A0022A07546 made by GENCI.

\section{References}

[1] N. Bassou and Y. Rharbi. "Role of Bénard-Marangoni Instabilities during Solvent Evaporation in Polymer Surface Corrugations". In: Langmuir 25.1 (2009), pp. 624-632.

[2] A. Batoul, H. Khallouf, and G. Labrosse. "A direct spectral solver of the 2D/3D unsteady Stokes problem. Application to the 2D square driven cavity". In: Comptes rendus de l'Académie des sciences. Série II, Mécanique, physique, chimie, astronomie 319.12 (1994), pp. 1455-1461.

[3] L. Baudey-Laubier. "Modélisation et simulation numérique des transferts de masse et de chaleur induits par évaporation". PhD thesis. Université Paris-Est, 2016.

[4] L. M. Blair and J. A. Quinn. "The onset of cellular convection in a fluid layer with time-dependent density gradients". In: Journal of Fluid Mechanics 36 (02 Apr. 1969), pp. 385-400.

[5] E. Chénier, C. Desceliers, C. Delcarte, B. Trouette, F. Doumenc, and B. Guerrier. "Sensitivity of diffusive-convective transition to the initial conditions in a transient Bénard-Marangoni problem". In: Proceedings of the International Heat Transfer Conference. 2010.

[6] P. Colinet, L. Joannes, C. Iorio, B. Haute, M. Bestehorn, G. Lebon, and J. Legros. "Interfacial turbulence in evaporating liquids: Theory and preliminary results of the ITEL-master 9 sounding rocket experiment". In: Advances in Space Research 32.2 (2003), pp. $119-127$.

[7] F. Doumenc, T. Boeck, B. Guerrier, and M. Rossi. "Transient Rayleigh-Bénard-Marangoni Convection due to Evaporation : a Linear Non-normal Stability Analysis". In: Journal of Fluid Mechanics 648 (2010), pp. 521-539.

[8] F. Doumenc, E. Chénier, B. Trouette, T. Boeck, C. Delcarte, B. Guerrier, and M. Rossi. "Free convection in drying binary mixtures: solutal versus thermal instabilities". In: International Journal of Heat and Mass Transfer 63 (2013), pp. 336-350.

[9] K. Goda. "A multistep technique with implicit difference schemes for calculating two- or three-dimensional cavity flows". In: Journal of Computational Physics 30.1 (1979), pp. 76 -95.

[10] J. Guermond, P. Minev, and J. Shen. "An overview of projection methods for incompressible flows". In: Computer Methods in Applied Mechanics and Engineering 195.44 - 47 (2006), pp. 6011 -6045.

[11] E. Leriche and G. Labrosse. "High-Order Direct Stokes Solvers with or Without Temporal Splitting: Numerical Investigations of Their Comparative Properties". In: SIAM Journal on Scientific Computing 22.4 (2000), pp. 1386-1410.

[12] H. Machrafi, A. Rednikov, P. Colinet, and P. C. Dauby. "Bénard instabilities in a binary-liquid layer evaporating into an inert gas". In: Journal of Colloid and Interface Science 349.1 (2010), pp. 331 -353.

[13] H. Machrafi, A. Rednikov, P. Colinet, and P. C. Dauby. "Time-dependent Marangoni-Bénard instability of an evaporating binary-liquid layer including gas transients". In: Physics of Fluids (2013).

[14] H. Machrafi, A. Rednikov, P. Colinet, and P. C. Dauby. "Importance of wave-number dependence of Biot numbers in one-sided models of evaporative Marangoni instability: Horizontal layer and spherical droplet". In: Physical Review E (2015).

[15] H. Mancini and D. Maza. "Pattern formation without heating in an evaporative convection experiment". In: EPL (Europhysics Letters) 66.6 (2004), p. 812.

[16] D. Merkt and M. Bestehorn. "Bénard-Marangoni convection in a strongly evaporating fluid". In: Physica D: Nonlinear Phenomena 185.3-4 (2003), pp. 196 -208.

[17] J. R. A. Pearson. "On convection cells induced by surface tension". In: Journal of Fluid Mechanics 4 (05 Sept. 1958), pp. $489-500$.

[18] O. Touazi, E. Chénier, F. Doumenc, and B. Guerrier. "Simulation of transient Rayleigh-Bénard-Marangoni convection induced by evaporation". In: International Journal of Heat and Mass Transfer 53.4 (2010), pp. 656-664.

[19] G. Toussaint, H. Bodiguel, F. Doumenc, B. Guerrier, and C. Allain. "Experimental characterization of buoyancy- and surface tension-driven convection during the drying of a polymer solution". In: International Journal of Heat and Mass Transfer 51.17-18 (2008), pp. $4228-4237$.

[20] B. Trouette, E. Chénier, C. Delcarte, and B. Guerrier. "Numerical study of convection induced by evaporation in cylindrical geometry". In: Eur. Phys. J. Special Topics 192 (1 2011), pp. 83-93.

[21] B. Trouette, E. Chénier, F. Doumenc, C. Delcarte, and B. Guerrier. "Transient Rayleigh-Bénard-Marangoni solutal convection". In: Physics of Fluids 24.7, 074108 (2012), pp. 1-25.

[22] J. Wang, G. Liu, Y. Fang, and W. Li. "Marangoni effect in nonequilibrium multiphase system of material processing". In: Reviews in Chemical Engineering (2016).

[23] S. G. Yiantsios, S. K. Serpetsi, F. Doumenc, and B. Guerrier. "Surface deformation and film corrugation during drying of polymer solutions induced by Marangoni phenomena". In: International Journal of Heat and Mass Transfer (2015). 